\title{
A Frame Bundle Generalization of Multisymplectic Momentum Mappings *†
}

\author{
J. K. Lawson \\ Department of Mathematics, Trinity University, 715 Stadium Drive, \\ San Antonio, TX 78212-7200 (e-mail: jlawson@trinity.edu)
}

\begin{abstract}
This paper presents generalized momentum mappings for covariant Hamiltonian field theories. The new momentum mappings arise from a generalization of symplectic geometry to $L_{V} Y$, the bundle of vertically adapted linear frames over the bundle of field configurations $Y$. Specifically, the generalized field momentum observables are vector-valued momentum mappings on the vertically adapted frame bundle generated from automorphisms of $Y$. The generalized symplectic geometry on $L_{V} Y$ is a covering theory for multisymplectic geometry on the multiphase space $Z$, and it follows that the field momentum observables on $Z$ are generalized by those on $L_{V} Y$. Furthermore, momentum observables on $L_{V} Y$ produce conserved quantities along flows in $L_{V} Y$. For translational and orthogonal symmetries of fields and reparametrization symmetry in mechanics, momentum is conserved, and for angular momentum in time-evolution mechanics we produce a version of the parallel axis theorem of rotational dynamics, and in special relativity, we produce the transformation of angular momentum under boosts.
\end{abstract}

\section{Contents}

\begin{tabular}{lll}
\hline 1 & Introduction & 2
\end{tabular}

2 Momentum mappings in multisymplectic geometry 3

3 Momentum mappings on the vertically adapted linear frame bundle $\quad 6$

4 Generalizing multisymplectic momentum mappings 10

$\begin{array}{lll}5 & \text { Conserved quantities } & 13\end{array}$

*2000 MSC: 53 D 20, 70 G 45, 57 R 15, 53 C 15, 37 J 15

${ }^{\dagger}$ Keywords: multisymplectic geometry, frame bundle, covariant field theories, Poisson bracket, momentum mappings. 
6 Examples $\quad 15$

6.1 Linear momentum in field theories . . . . . . . . . . . . . . . . 15

6.2 Angular momentum in field theories . . . . . . . . . . . . . . . 16

6.3 Affine symmetry in time-evolution mechanics . . . . . . . . . . . . . . 18

6.4 Time reparametrization symmetry in time-evolution mechanic . . . . . . . 20

\begin{tabular}{llr}
\hline Conclusions & 21
\end{tabular}

\section{Introduction}

Norris' generalization [15, 16, 17] of the symplectic geometry of the cotangent bundle has been a successful theoretical tool for particle mechanics. Norris has shown that the standard symplectic geometry of the cotangent bundle to an $n$-dimensional manifold may be obtained in its entirety from the $n$-symplectic geometry of the linear frame bundle. The key component of $n$-symplectic geometry is recognizing that the canonical soldering one-form of a frame bundle [9] may be employed as a vector-valued $n$-symplectic potential.

Subsequently, it has been shown [4, 10] that the multisymplectic geometry of the (affine) multiphase space $Z$ introduced by Kijowski [7, 8] and refined by Gotay, et al. [6], may be generalized by adapting Norris's theory to $L_{V} Y$, the bundle of vertically adapted linear frames of a configuration bundle $Y$ of a classical field. We may use the new theory to reproduce the Poisson bracket of momentum observables on $Z$. However, the momentum observables on $Z$ are not closed under this bracket, whereas the analogous momentum observables on $L_{V} Y$ are closed under the analogous Poisson bracket [4, 10].

The purpose of this paper is to extend this work in generalized symplectic geometry to include momentum mappings for field theories. In the Hamiltonian framework, momentum mappings are the foundation for obtaining conserved quantities from group symmetries of phase space (the symplectic or multisymplectic manifold). Because the generalized symplectic structure is invariant under automorphisms of $Y$ lifted to $L_{V} Y$, momentum mappings on $L_{V} Y$ induce the momentum mappings on $Z$ found in [6]. This is analogous to the result of Norris [16], which induces the momentum mappings of standard symplectic geometry of the cotangent bundle from the theory of $n$-symplectic geometry. The momentum observables on $L_{V} Y$ established in [4] and [10] are shown to be a special case of the new momentum mappings obtained from the Lie algebra of projectable vector fields on $Y$.

When we consider momentum mappings constructed from the infinitesimal generators of the translation group, an orthogonal group, or an affine group, we obtain conserved quantities along flows that give us "frame bundle" versions of conservation of linear field momentum, conservation of angular field momentum, and a "parallel axis theorem" for field theories. We may also use time-reparametrization symmetry in mechanics to extend an application of Norris [15, 17] regarding parallel transport of frames along geodesics of a Riemannian metric.

The format of this paper is as follows. First we summarize multisymplectic geometry in Section 2, and then we introduce momentum mappings on $L_{V} Y$ in Section 3. In Section 4 we generate momentum mappings on $Z$ from those on $L_{V} Y$, and in Section 5 we derive conserved quantities. Examples are found in Section 6. 


\section{Momentum mappings in multisymplectic geometry}

Let $X$ be an oriented $n$-dimensional manifold and let $\pi_{X Y}: Y \rightarrow X$ be a fiber bundle with standard fiber a $k$-dimensional manifold. (Note: In general, we shall denote a projection from $A$ onto $B$ as $\pi_{B A}$.) A classical field is a section of the field configuration space $Y$ over the parameter space $X$. From local coordinates $\left\{x^{i}\right\}, i=1, \ldots, n$ on $X$ we may construct local adapted coordinates $\left\{x^{i}, y^{A}\right\}, i=1, \ldots, n, A=1, \ldots, k$, on $Y$. The multivelocity bundle is the (first-order) jet bundle $J Y$, the affine bundle over $Y$ whose fiber over $y \in Y$ consists of linear maps $\gamma_{y}: T_{\pi_{X Y}(y)} X \rightarrow T_{y} Y$ satisfying $\pi_{X Y *} \circ \gamma_{y}=\operatorname{Id}_{T_{\pi_{X Y}(y)} X}$. A section of $J Y$ over $Y$ can be identified with an Ehresmann connection on $\pi_{X Y}: Y \rightarrow X$.

The bundle of affine cojets [5, 6] $J^{\star} Y$ is the vector bundle over $Y$ whose fiber at $y$ is the set of affine maps from $J_{y} Y$ to $\wedge_{\pi_{X Y}(y)}^{n} X$. It follows that $\operatorname{dim} J^{\star} Y=\operatorname{dim} J Y+1$. An equivalent description [6] of $J^{\star} Y$ is useful. Define the vertical subbundle of $T Y$ to be

$$
V(T Y):=\left\{w_{y} \mid y \in Y, w_{y} \in T_{y} Y \text { and } \pi_{X Y *}\left(w_{y}\right)=0\right\} .
$$

The multiphase space $Z$ [6, 8] is the fiber bundle whose fiber $Z_{y}$ over $y \in Y$ is

$$
\left.\left.Z_{y}:=\left\{z \in \wedge_{y}^{n} Y \mid v\right\lrcorner w\right\lrcorner z=0 \forall v, w \in V\left(T_{y} Y\right)\right\},
$$

where $\downarrow$ denotes the inner product of a vector with a differential form. The bundle $Z$, originally defined by Kijowski [8], admits a canonical $n$-form,

$$
\Theta(z)=\pi_{Y Z}^{*}(z)
$$

which is the pullback via inclusion $Z \hookrightarrow \wedge^{n} Y$ of the canonical $n$-form on $\wedge^{n} Y$, and $Z$ is "canonically" isomorphic to $J^{\star} Y$ [6]. We can define coordinates $\left\{x^{i}, y^{A}, p_{B}^{j}, p\right\}$ on $Z$ where $\left\{x^{i}, y^{A}\right\}$ are the lifts of the adapted coordinates of $Y$,

$$
\begin{aligned}
p(z) & \left.\left.\left.=\frac{\partial}{\partial x^{n}}\right\lrcorner \cdots\right\lrcorner \frac{\partial}{\partial x^{1}}\right\lrcorner z, \quad \text { and } \\
p_{B}^{j}(z) & \left.\left.\left.=(-1)^{j-1} \frac{\partial}{\partial x^{n}}\right\lrcorner \frac{\partial}{\partial x^{n-1}}\right\lrcorner \ldots \downarrow \frac{\widehat{\partial}}{\partial x^{j}}\right\lrcorner \ldots \downarrow \frac{\partial}{\partial x^{1}} \downarrow \frac{\partial}{\partial y^{B}} \downarrow z,
\end{aligned}
$$

where $\frac{\widehat{\partial}}{\partial x^{j}}$ denotes the omission of $\frac{\partial}{\partial x^{j}}$. If we define in local lifted coordinates

$$
\left.\left.\left.d^{n} x:=d x^{1} \wedge \cdots \wedge d x^{n}, \quad d^{n-1} x_{i}:=\frac{\partial}{\partial x^{i}}\right\lrcorner d^{n} x, \text { and } d^{n-2} x_{i j}:=\frac{\partial}{\partial x^{j}}\right\lrcorner \frac{\partial}{\partial x^{i}}\right\lrcorner d^{n} x,
$$

then $\Theta$ may be expressed locally as

$$
\Theta=p_{A}^{i} d y^{A} \wedge d^{n-1} x_{i}+p d^{n} x
$$

The $(n+1)-$ form $d \Theta$ is nondegenerate and thus may be considered a multisymplectic structure form on $Z$. The pair $(Z, d \Theta)$ is called a multisymplectic manifold [6].

Definition [5, 6] Let a Lie group $\mathcal{G}$ act on the left on $Z$ and the multisymplectic structure form $d \Theta$ be invariant under this action. Let $\mathfrak{g}$ be the Lie algebra of $\mathcal{G}$. A mapping

$$
J: Z \rightarrow \mathfrak{g}^{*} \otimes \wedge^{n-1} Z
$$


is a momentum mapping if $J$ covers the identity on $Z$ and if $\hat{J}$ is defined by

$$
\hat{J}(\xi)(z)=\langle J(z), \xi\rangle
$$

then

$$
\left.d \hat{J}(\xi)=-\xi_{Z}\right\lrcorner d \Theta
$$

where $\xi_{Z}$ is the infinitesimal generator of the $\mathcal{G}$-action on $Z$ induced by $\xi \in \mathfrak{g}$.

Definition A Hamiltonian observable on $Z$ is an $(n-1)$-form $f$ on $Z$ that satisfies

$$
d f=-X\lrcorner d \Theta
$$

for some vector field $X$ on $Z$. The vector field $X$ is called a Hamiltonian vector field on $Z$.

For a momentum mapping $J$ and $\xi \in \mathfrak{g}, \hat{J}(\xi)$ is a Hamiltonian observable and $\xi_{Z}$ is the corresponding Hamiltonian vector field.

The group Aut $Y$ of fiber bundle automorphisms of $Y$ over $X$ is the multisymplectic analogue of the group Diff $X$ of diffeomorphisms of configuration space in the symplectic geometry of the cotangent bundle $T^{*} X$. Let $\mathcal{X} Y$ be the Lie algebra of vector fields on $Y$. Denote the space of vector fields of $Y$ projectable to $X$ by $\mathcal{X}_{\text {Proj }} Y$. Note that $\mathcal{X}_{\text {Proj }} Y$ is a Lie subalgebra of $\mathcal{X} Y$, since $\left[\pi_{X Y *} v, \pi_{X Y *} w\right]=\pi_{X Y *}[v, w]$ [3, p. 85].

Lemma 2.1 The group Aut $Y$ formally has for its Lie algebra the vector space of complete projectable vector fields on $Y$.

Proof (Motivated by [1].) We identify the Lie algebra of Aut $Y$ with the set of all vector fields of $Y$ which are tangents to curves through the identity of Aut $Y$. Let $\left\{\eta_{Y_{\lambda}} \mid \lambda \in \mathbb{R}\right\}$ be a curve in Aut $Y$ such that $\eta_{Y_{0}}=\operatorname{Id}_{Y}$. For each $\eta_{Y \lambda}$ there exists $\eta_{X \lambda}$, a diffeomorphism of $X$ such that $\pi_{X Y} \circ \eta_{Y \lambda}=\eta_{X \lambda} \circ \pi_{X Y}$. Since $\eta_{Y \lambda} \in$ Aut $Y \subset \operatorname{Diff} Y, v:=\frac{d}{d \lambda} \eta_{Y \lambda} \mid{ }_{\lambda=0}$ is a tangent vector at $\operatorname{Id}_{Y}, \tau_{Y} \circ v=\operatorname{Id}_{X}$ and

$$
\pi_{X Y *} \circ v(y)=\left.\frac{d}{d \lambda}\left(\pi_{X Y} \circ \eta_{\lambda}(y)\right)\right|_{\lambda=0}=\left.\frac{d}{d \lambda} \eta_{X \lambda}\right|_{\lambda=0}\left(\pi_{X Y}(y)\right)=: \underline{v} \circ \pi_{X Y}(y) .
$$

Observe that if $\pi_{X T X}: T X \rightarrow X$ is projection then $\pi_{X T X} \circ \underline{v}=\operatorname{Id}_{X}$. Therefore, $T_{\operatorname{Id}_{Y}} \operatorname{Aut} Y$ is isomorphic to a vector subspace of $\mathcal{X}_{\mathrm{Proj}} Y$. In the case that $Y$ is compact we shall show that $T_{\mathrm{Id} Y}$ Aut $Y$ is all of the space $\mathcal{X}_{\mathrm{Proj}} Y$. Let $\phi$ be the flow box [1] for $v$ at $y$ and let $\underline{\Phi}$ be the flow box for $\underline{v}$ at $\pi_{X Y}(y)$. Then for each $y \in Y$, we have that $\pi_{X Y} \circ \Phi_{0}(y)=\underline{\Phi}_{0} \circ \pi_{X Y}(y)$,

$$
\frac{d}{d t}\left(\pi_{X Y} \circ \Phi_{t}\right)(y)=\pi_{X Y *} v\left(\Phi_{t}(y)\right)=\underline{v}\left(\pi_{X Y}\left(\Phi_{t}(y)\right)\right)=\underline{v}\left(\left(\pi_{X Y} \circ \Phi_{t}\right)(y)\right)
$$

and

$$
\frac{d}{d t}\left(\underline{\Phi}_{t} \circ \pi_{X Y}\right)(y)=\underline{v}\left(\underline{\Phi}_{t}\left(\pi_{X Y}(y)\right)\right)=\underline{v}\left(\left(\underline{\Phi}_{t} \circ \pi_{X Y}\right)(y)\right) .
$$

Thus $\pi_{X Y} \circ \Phi_{t}(y)$ and $\underline{\Phi}_{t} \circ \pi_{X Y}(y)$ are curves in $X$ that solve the same initial value problem on open neighborhoods of $t$ and of $\pi_{X Y}(y)$. By an existence and uniqueness theorem [1], 
they are equal. Therefore $\pi_{X Y} \circ \Phi_{t}=\underline{\Phi}_{t} \circ \pi_{X Y}$ so the projectable vector field $v$ defines a one-parameter curve $\left\{\Phi_{t} \mid t \in \mathbb{R}\right\}$ in Aut $Y$ such that $v=\left.\frac{d}{d t} \Phi_{t}\right|_{t=0}$.

We shall formally identify $\mathcal{X} Y$ with the tangent space at the identity of Diff $Y$, the group of diffeomorphisms of $Y$. Subsequently, we will formally identify $\mathcal{X}_{\text {Proj }} Y$ with the tangent space at the identity of Aut $Y$. However this ignores the topology of $Y$. Milnor [13] notes that if $Y$ is compact then Diff $Y$ is a Lie group that admits a $C^{\infty}$ manifold structure. For noncompact $Y$ we may choose only to consider diffeomorphisms which are the identity outside a compact subset of $Y$. This leads to vector fields that vanish outside this subset.

There is a slight ambiguity because $\mathcal{X}_{\text {Proj }} Y$ serves both as the Lie algebra and as the collection of infinitesimal generators. To resolve this, let $[\xi, \zeta]$ denote the Lie bracket defined on the formal Lie algebra of left invariant vector fields on the manifold Aut $Y$, and let $\left[\xi_{Y}, \zeta_{Y}\right]$ denote the usual Lie bracket defined on $\mathcal{X} Y$ (in which the vector fields are right invariant with respect to the Aut $Y$ action). See [1], Exercise 4.1G] for a clarification.

The canonical lift [6] of $\eta_{Y} \in$ Aut $Y$ is a map

$$
\eta_{Z}: Z \rightarrow Z: z \mapsto\left(\eta_{Y}^{-1}\right)^{*}(z)
$$

The map $\eta_{Z}$ is a $\pi_{X Z}$-bundle map under which $\Theta$ is invariant. For $\xi \in \mathcal{X}_{\text {Proj }} Y$, it follows that $\mathbf{L}_{\xi_{Z}} \Theta=0$ and the induced momentum mapping defines the momentum observable

$$
\left.\left.\hat{J}(\xi)(z):=\xi_{Z}\right\lrcorner \Theta(z)=\pi_{Y Z}^{*}\left(\xi_{Y}\right\lrcorner z\right),
$$

where $\xi_{Y}$ is the infinitesimal generator of the ensuing action of Aut $Y$ on $Y$. It follows that $\hat{J}$ is $A d^{*}$-equivariant. That is, $J\left(A d_{\eta}^{-1} \xi\right)=\eta_{Z}^{*}(\hat{J}(\xi))$. Let $T^{1}(Z)$ denote the vector space of momentum observables, and observe that $\xi \mapsto \hat{J}(\xi)$ is a bijection from $\mathcal{X}_{\text {Proj }} Y$ to $T^{1}(Z)$. then

If in local adapted coordinates on we write $\xi \in \mathcal{X}_{\text {Proj }} Y$ as $\xi=\xi^{i}\left(x^{j}\right) \frac{\partial}{\partial x^{i}}+\xi^{A}\left(x^{j}, y^{B}\right) \frac{\partial}{\partial y^{A}}$,

$$
\hat{J}(\xi)(z)=\left(p_{A}^{i} \xi^{A}+p \xi^{i}\right) d^{n-1} x_{i}-p_{A}^{i} \xi^{j} d y^{A} \wedge d^{n-2} x_{i j}
$$

and

$$
\xi_{Z}=\xi^{k} \frac{\partial}{\partial x^{k}}+\xi^{A} \frac{\partial}{\partial y^{A}}+\left(p_{A}^{j} \frac{\partial \xi^{i}}{\partial x^{j}}-p_{A}^{i} \frac{\partial \xi^{j}}{\partial x^{j}}-p_{B}^{i} \frac{\partial \xi^{B}}{\partial y^{A}}\right) \frac{\partial}{\partial p_{A}^{i}}-\left(p \frac{\partial \xi^{i}}{\partial x^{i}}+p_{A}^{i} \frac{\partial \xi^{A}}{\partial x^{i}}\right) \frac{\partial}{\partial p} .
$$

From (2.5) if $\xi_{Y}, \zeta_{Y} \in \mathcal{X}_{\mathrm{Proj}} Y$ then $\pi_{Y Z *}\left[\xi_{Z}, \zeta_{Z}\right]=\left[\xi_{Y}, \zeta_{Y}\right]$, and thus, by (2.3),

$$
\left.\left.\left[\xi_{Z}, \zeta_{Z}\right]\right\lrcorner \Theta_{z}=\pi_{Y Z}^{*}\left(\left[\xi_{Y}, \zeta_{Y}\right]\right\lrcorner z\right)
$$

If we define a Poisson bracket by

$$
\{\hat{J}(\xi), \hat{J}(\zeta)\}:=-d \Theta\left(\xi_{Z}, \zeta_{Z}\right)
$$

then using $\mathbf{L}_{\xi_{Z}} \Theta=0$ and the Lie derivative identities [1, p. 121],

$$
[X, Y] \boldsymbol{\lrcorner} \alpha=\mathbf{L}_{X}(Y \boldsymbol{\lrcorner} \alpha)-Y \boldsymbol{\lrcorner}\left(\mathbf{L}_{X} \alpha\right) \quad \text { and } \quad \mathbf{L}_{X} \alpha=X \boldsymbol{\lrcorner} d \alpha+d(X \boldsymbol{\lrcorner} \alpha) \text {, }
$$


we obtain

$$
\left.\left.\{\hat{J}(\xi), \hat{J}(\zeta)\}=\hat{J}([\xi, \zeta])-d\left(\xi_{Z}\right\lrcorner \zeta_{Z}\right\lrcorner \Theta\right)
$$

For an alternative proof using the $\mathrm{Ad}^{*}$ equivariance of $J$, see [6, Prop. 4.5].

The Poisson bracket on $T^{1}(Z)$ given by (2.7) is not a true Poisson bracket because there lacks an associative multiplication of $(n-1)$-forms on which the bracket acts as a derivation. Worse, $\left.\left.d\left(\xi_{Z}\right\lrcorner \zeta_{Z}\right\lrcorner \Theta\right)$ is not in $T^{1}(Z)$ because from equation (2.2) the Hamiltonian vector field of an exact form is the zero vector field on $Z$, but the momentum observable corresponding to the zero vector field is the zero $(n-1)$-form on $Z$. Thus, $T^{1}(Z)$ is not closed under the Poisson bracket. Equation (2.9) explains the remark in [6] that the Poisson bracket of two momentum observables "is up to the addition of exact terms, another momentum observable."

\section{Momentum mappings on the vertically adapted linear frame bundle}

For an $n$-dimensional manifold $M$, the linear frame bundle $L M$ has an $\mathbb{R}^{n}$-valued canonical soldering one-form [9], which we regard as an $n$-symplectic potential. This geometric object has been shown [16] to generate the canonical one-form on the cotangent bundle $T^{*} M$. Norris's program of $n$-symplectic geometry [15, 16, 17] originates from the exterior derivative of the soldering form, which is a closed nondegenerate two-form serving as an $\mathbb{R}^{n}$-valued $n$ symplectic structure form. The $n$-symplectic geometry generalizes the symplectic geometry of classical particle mechanics on $T^{*} M$.

Now, for the $(n+k)$-dimensional field configuration bundle $Y$, we shall treat the canonical soldering form $\theta$ on $L Y$ as an $(n+k)$-symplectic potential $\theta$.

Definition (motivated by the definition of an adapted frame [9]) The vertically adapted frame bundle $L_{V} Y$ is defined to be

$$
L_{V} Y:=\left\{\left(y,\left\{e_{i}, \epsilon_{A}\right\}\right) \in L Y \mid\left\{\epsilon_{A}\right\} \text { is a frame of } V\left(T_{y} Y\right)\right\} .
$$

The bundle $L_{V} Y$ is a reduced subbundle of $L Y$ obtained by breaking the $G L(n+k)$ symmetry of $L Y$ [10].

The structure group of $L_{V} Y$, expressed with respect to the standard basis of $\mathbb{R}^{n+k}$ is the adapted linear group

$$
G_{\mathcal{A}}:=\left\{\left(\begin{array}{cc}
N & 0 \\
A & K
\end{array}\right) \mid N \in G L(n), K \in G L(k), A \in \mathbb{R}^{k \times n}\right\}
$$

with multiplication defined by block matrix multiplication. For convenience we write $\left(\begin{array}{cc}N & 0 \\ A & K\end{array}\right) \in G_{\mathcal{A}}$ as $(N, K, A)$. The free right action of $G_{\mathcal{A}}$ on $L_{V} Y$ is given by

$$
\left(y,\left\{e_{i}, \epsilon_{A}\right\}\right) \cdot(N, K, A):=\left(y,\left\{e_{j} N_{i}^{j}+\epsilon_{B} A_{i}^{B}, \epsilon_{B} K_{A}^{B}\right\}\right)
$$


Let $\left(y,\left\{e^{i}, \epsilon^{A}\right\}\right)$ be the coframe dual to the vertically adapted frame $\left(y,\left\{e_{i}, \epsilon_{A}\right\}\right)$. We may define the right action of $G_{\mathcal{A}}$ on $\left(y,\left\{e^{i}, \epsilon^{A}\right\}\right)$ by

$$
\left(y,\left\{e^{i}, \epsilon^{A}\right\}\right) \cdot(N, K, A)=\left(y,\left\{\left(N^{-1}\right)_{j}^{i} e^{j},-\left(K^{-1} A N^{-1}\right)_{j}^{A} e^{j}+\left(K^{-1}\right)_{B}^{A} \epsilon^{B}\right\}\right) .
$$

The coframe in (3.2) is dual to the frame $\left(y,\left\{e_{i}, \epsilon_{A}\right\}\right) \cdot(N, K, A)$.

The pullback of the $(n+k)$-symplectic structure form $d \theta$ via inclusion $i: L_{V} Y \hookrightarrow L Y$ is closed and nondegenerate on $L_{V} Y$, just as $d \theta$ is on $L Y$. Local coordinates on $L_{V} Y$ are $\left\{x^{i}, y^{A}, \pi_{j}^{i}, \pi_{B}^{A}, \pi_{i}^{A}\right\}$, where $\left\{x^{i}, y^{A}\right\}$ are local adapted coordinates on $Y$ and $\pi_{j}^{i}:=e^{i}\left(\frac{\partial}{\partial x^{j}}\right)$, $\pi_{B}^{A}:=\epsilon^{A}\left(\frac{\partial}{\partial y^{B}}\right)$ and $\pi_{j}^{A}:=\epsilon^{A}\left(\frac{\partial}{\partial x^{j}}\right)$. Let $\left\{R_{\mu}\right\}_{\mu=1,2, \ldots, n+k}$ be the standard basis of $\mathbb{R}^{n+k}$, $\left\{r_{i}\right\}_{i=1,2, \ldots, n}$ be the standard basis of $\mathbb{R}^{n}$, and $\left\{s_{A}\right\}_{A=1,2, \ldots, k}$ be the standard basis of $\mathbb{R}^{k}$. Then, we define $\hat{r}_{i}:=\left(r_{i}, 0\right) \in \mathbb{R}^{n+k}$ and $\hat{s}_{A}:=\left(0, s_{A}\right) \in \mathbb{R}^{n+k}$. Thus, in local coordinates,

$$
i^{*} \theta=\pi_{j}^{i} d x^{j} \hat{r}_{i}+\left(\pi_{i}^{A} d x^{i}+\pi_{B}^{A} d y^{B}\right) \hat{s}_{A}
$$

and

$$
i^{*} d \theta=d \pi_{j}^{i} \wedge d x^{j} \otimes \hat{r}_{i}+\left(d \pi_{i}^{A} \wedge d x^{i}+d \pi_{B}^{A} \wedge d y^{B}\right) \otimes \hat{s}_{A}
$$

Definition A Hamiltonian observable on $L_{V} Y$ is an $\mathbb{R}^{n}$-valued function $\hat{f}$ on $L_{V} Y$ that satisfies

$$
\left.d \hat{f}=-X_{\hat{f}}\right\lrcorner i^{*} d \theta
$$

for some $X \in \mathcal{X}\left(L_{V} Y\right)$. The vector field $X_{\hat{f}}$ is then called a Hamiltonian vector field on $L_{V} Y$.

As in the $n$-symplectic theory on $L M$ [15, 16, 17], equation (3.4) admits neither all vector fields nor all $\mathbb{R}^{n+k}$-valued functions.

\section{Definitions}

- The vector space of Hamiltonian observables on $L_{V} Y$ is denoted by $H F^{1}\left(L_{V} Y\right)$.

- The vector space of Hamiltonian vector fields on $L_{V} Y$ is denoted by $H V^{1}\left(L_{V} Y\right)$.

- $T^{1}\left(L_{V} Y\right)$ is the vector space of tensorial $\mathbb{R}^{n+k}$-valued functions on $L_{V} Y$.

An element of $T^{1}\left(L_{V} Y\right)$ can be expressed in local coordinates as

$$
\hat{f}=f^{i}\left(x^{k}, y^{C}\right) \pi_{i}^{j} \otimes \hat{r}_{j}+f^{A}\left(x^{k}, y^{C}\right) \pi_{A}^{B} \otimes \hat{s}_{B}+f^{i}\left(x^{k}, y^{C}\right) \pi_{i}^{B} \otimes \hat{s}_{B} .
$$

For $\hat{f} \in T^{1}\left(L_{V} Y\right)$ we solve equation (3.4) locally for $X_{\hat{f}}$. This yields

$$
X_{\hat{f}}=f^{i} \frac{\partial}{\partial x^{i}}+f^{A} \frac{\partial}{\partial y^{A}}-\frac{\partial f^{k}}{\partial x^{j}} \pi_{k}^{i} \frac{\partial}{\partial \pi_{j}^{i}}-\frac{\partial f^{C}}{\partial y^{B}} \pi_{C}^{A} \frac{\partial}{\partial \pi_{B}^{A}}-\left(\frac{\partial f^{j}}{\partial x^{i}} \pi_{j}^{A}+\frac{\partial f^{B}}{\partial x^{i}} \pi_{B}^{A}\right) \frac{\partial}{\partial \pi_{i}^{A}},
$$


subject to the constraints on $\hat{f}$,

$$
\frac{\partial f^{i}}{\partial y^{A}}=0 \quad \forall i=1, \ldots, n \text { and } A=1, \ldots, k .
$$

Thus, $T^{1}\left(L_{V} Y\right) \not \subset H F^{1}\left(L_{V} Y\right)$. Define $T_{V}^{1}\left(L_{V} Y\right):=H F^{1}\left(L_{V} Y\right) \cap T^{1}\left(L_{V} Y\right)$. Since $L_{V} Y$ is a subbundle of $L Y$, a point $w \in L_{V} Y$ is a linear isomorphism [9] $w: \mathbb{R}^{n+k} \rightarrow T_{\pi_{Y_{L_{V}}(}(w)} Y$. Moreover, for $g \in G_{\mathcal{A}},(w \cdot g) V=w(g V)$ for each $V \in \mathbb{R}^{n+k}$. Hence a tensorial function $\hat{f}$ corresponds bijectively to $\mathbf{f} \in \mathcal{X} Y$ by the relation $\hat{f}(w)=w^{-1}\left(\mathbf{f}\left(\pi_{Y L_{V} Y}(w)\right)\right)$. Now, if $\hat{f} \in T_{V}^{1}\left(L_{V} Y\right)$ then, by (3.5), $f^{i}=f^{i}\left(x^{j}\right)$ and $\hat{f}$ is induced from a projectable vector field. Conversely, $\mathbf{f} \in \mathcal{X}_{\text {Proj }} Y$ gives a tensorial function $\hat{f}$ satisfying (3.5). Combine this with the results of Section 2 to obtain the following proposition [10].

Proposition $3.1 T_{V}^{1}\left(L_{V} Y\right), T^{1}(Z)$, and $\mathcal{X}_{\text {Proj }} Y$ are in pairwise bijective correspondence. Furthermore, if $n \geq 2$ and $k \geq 2$ then $H F^{1}\left(L_{V} Y\right) \simeq T_{V}^{1}\left(L_{V} Y\right) \oplus C^{\infty}\left(X, \mathbb{R}^{n}\right) \oplus C^{\infty}\left(Y, \mathbb{R}^{k}\right)$.

Definition Let $\mathcal{G}$ be a Lie group with an action on $L_{V} Y$ under which $i^{*} d \theta$ is invariant. Let $\mathfrak{g}$ be the Lie algebra of $\mathcal{G}$. A mapping $J: L_{V} Y \rightarrow \mathfrak{g}^{*} \otimes \mathbb{R}^{n+k}$ is a momentum mapping if

$$
\left.d \hat{J}(\xi)=-\xi_{L_{V} Y}\right\lrcorner d i^{*} \theta \quad \forall \xi \in \mathfrak{g}
$$

where $\xi_{L_{V} Y}$ is the infinitesimal generator of the $\mathcal{G}$-action on $L_{V} Y$ generated by $\xi \in \mathfrak{g}$ and $\hat{J}(\xi): L_{V} Y \rightarrow \mathbb{R}^{n+k}$ is defined by

$$
\hat{J}(\xi)(w)=<J(w), \xi>\quad \forall w \in L_{V} Y .
$$

Note that a momentum mapping on $L_{V} Y$ is the restriction of a momentum mapping on $L Y$ (as defined in [16]). When necessary, we will write $J=J_{L_{V} Y}$ to clarify that $J$ is a momentum mapping on $L_{V} Y$. Furthermore, for any $\xi \in \mathfrak{g}, \hat{J}(\xi) \in H F^{1}\left(L_{V} Y\right)$ and its corresponding Hamiltonian vector field $X \in H V^{1}\left(L_{V} Y\right)$.

Now we consider momentum mappings when $\mathcal{G}=\operatorname{Aut} Y$. Define $\operatorname{Aut}\left(L_{V} Y\right)$ to be the subgroup of $\operatorname{Diff}\left(L_{V} Y\right)$ whose elements are fiber bundle automorphisms both over $Y$ and over $X$. Let $\eta_{Y} \in$ Aut $Y$ cover $\eta_{X} \in \operatorname{Diff} X$. Define the mapping

$$
\eta_{L_{V} Y}: L_{V} Y \rightarrow L_{V} Y:\left(y,\left\{e_{i}, \epsilon_{A}\right\}\right) \mapsto\left(\eta_{Y}(y),\left\{\eta_{Y *} e_{i}, \eta_{Y *} \epsilon_{A}\right\}\right)
$$

Clearly, $\eta_{L_{V} Y}$ is bijective and smooth and has a smooth inverse. By definition, $\pi_{Y L_{V} Y}$ ○ $\eta_{L_{V} Y}=\eta_{Y} \circ \pi_{Y L_{V} Y}$. Since $\pi_{X Y *} \eta_{Y *} \epsilon_{A}=\eta_{X *} \pi_{X Y *} \epsilon_{A}=0$, it follows that $\eta_{Y *} \epsilon_{A} \epsilon$ $V\left(T_{\eta_{Y}(y)} Y\right)$ and thus $\eta_{L_{V} Y} \in \operatorname{Aut}\left(L_{V} Y\right)$. Therefore, $\eta_{L_{V} Y}$ is the canonical lift of $\eta_{Y}$.

Remark We cannot naturally define a lift of Diff $Y$ to $\operatorname{Aut}\left(L_{V} Y\right)$. Indeed, let $X=\mathbb{R}$ and $Y=X \times \mathbb{R}$. Define $f \in \operatorname{Diff} Y$ by $f(x, y)=(y, x)$. Then $f_{*}\left(\frac{\partial}{\partial y}\right)=\frac{\partial}{\partial x}$, which implies that $f_{*}(V(T Y)) \nsubseteq V(T Y)$.

Proposition 3.2 The $(n+k)$-symplectic potential $i^{*} \theta$ on $L_{V} Y$ is invariant under the canonical lift of each automorphism of $Y$. Conversely, every element of $\operatorname{Aut}\left(L_{V} Y\right)$ that leaves $i^{*} \theta$ invariant is a lift of an automorphism of $Y$. 
Proof We modify a result of Kobayashi and Nomizu [9]. Let $\eta_{Y} \in$ Aut $Y$ and let $\eta_{L_{V} Y}$

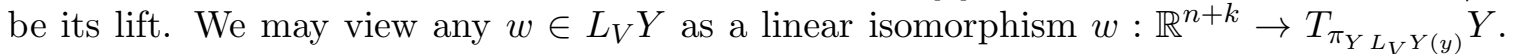
Then, as linear isomorphisms, $\eta_{L_{V} Y}(w)=\eta_{Y * w} \circ w$, and thus $\eta_{L_{V} Y}(w)^{-1} \circ \eta_{Y * w}=w^{-1}$. So, for $X_{w} \in T_{w}\left(L_{V} Y\right)$,

$$
\begin{aligned}
\eta_{L_{V} Y}^{*} i^{*} \theta\left(X_{w}\right) & =i^{*} \theta\left(\left(\eta_{L_{V} Y * X}\right)_{\eta_{L_{V} Y}(w)}\right) \\
& =\eta_{L_{V} Y}(w)^{-1}\left(\pi_{Y L_{V} Y *} \eta_{L_{V} Y * X}\right)_{\eta_{L_{V} Y}(w)} \\
& =\eta_{L_{V} Y}(w)^{-1} \circ \eta_{Y * w}\left(\pi_{Y L_{V} Y *} X\right) \\
& =w^{-1}\left(\pi_{Y L_{V} Y *} X\right) \\
& =i^{*} \theta\left(X_{w}\right) .
\end{aligned}
$$

Conversely, let $F \in \operatorname{Aut}\left(L_{V} Y\right)$ leave $i^{*} \theta$ invariant and cover $\eta_{Y} \in \operatorname{Aut} Y$. That is, $\pi_{Y L_{V} Y} \circ$ $F=\eta_{Y} \circ \pi_{Y L_{V} Y}$. Let $\tilde{\eta}_{L_{V} Y}$ denote the natural lift of $\eta_{Y}$ and define $H:=\tilde{\eta}_{L_{V} Y}^{-1} \circ F$. Then $H$ leaves $i^{*} \theta$ invariant, and $\pi_{Y L_{V} Y} \circ H=\pi_{Y L_{V} Y}$. So for $X_{w} \in T_{w}\left(L_{V} Y\right)$,

$$
\begin{aligned}
H^{*} i^{*} \theta\left(X_{w}\right) & =i^{*} \theta\left(X_{w}\right) \\
H(w)^{-1}\left(\pi_{Y L_{V}{ }_{*}} H_{*} X\right) & =w^{-1}\left(\pi_{Y L_{V} Y *} X_{w}\right) \\
H(w)^{-1}\left(\pi_{Y L_{V} Y *} X\right) & =w^{-1}\left(\pi_{Y L_{V} Y *} X\right)
\end{aligned}
$$

So $H(w)=w \forall w \in L_{V} Y$ and thus $H=\operatorname{Id}_{L_{V} Y}$.

Denote the action of Aut $Y$ on $Y$ by $\Psi$ : Aut $Y \times Y \rightarrow Y$. By Proposition 3.2, this action lifts to an $(n+k)$-symplectic action $\tilde{\Psi}:$ Aut $Y \times L_{V} Y \rightarrow L_{V} Y$. Now, a projectable vector field generates a one-parameter group $\psi_{t}$ of local automorphisms of $Y$, which lifts to a one-parameter group $\tilde{\psi}_{t}$ of elements of $\operatorname{Aut}(U)$ for some open set $U \subset L_{V} Y$. The vector field $\xi_{U}:=(d / d t) \tilde{\psi}_{t}$ on $U$ is the infinitesimal generator of $\tilde{\psi}_{t}$ and thus is the natural lift of $\xi_{\pi_{X Y}(U)}$

Lemma 3.3 Let $\xi \in \mathcal{X}_{\text {Proj }} Y$ be the infinitesimal generator of the action of Aut $Y$ on $L_{V} Y$. Then

$$
\left.\hat{J}(\xi):=\xi_{L_{V} Y}\right\lrcorner i^{*} \theta
$$

is in $T_{V}^{1}\left(L_{V} Y\right)$, and its corresponding momentum mapping $J$ is $\mathrm{Ad}^{*}$ equivariant. Furthermore, every element of $T_{V}^{1}\left(L_{V} Y\right)$ can be identified with $\hat{J}(\xi)$ for some $\xi \in \mathcal{X}_{\text {Proj }} Y$.

Proof Because $i^{*} \theta$ is invariant under $\tilde{\Psi}$, it follows that $\mathbf{L}_{\xi_{L_{V}} i} i^{*} \theta=0$. Using (2.8), we obtain $d\left(\xi_{L_{V} Y} \downarrow i^{*} \theta\right)=-\xi_{L_{V} Y} \downarrow i^{*} d \theta$, so $\xi_{L_{V} Y} \downarrow i^{*} \theta \in H F^{1}\left(L_{V} Y\right)$. If $\tilde{\psi}_{t}$ is the local flow of $\xi_{L_{V} Y}$ in a neighborhood of $w \in L_{V} Y$, then for $g \in G_{\mathcal{A}}, R_{g} \circ \tilde{\psi}_{t}=\tilde{\psi}_{t} \circ R_{g}$ since $\tilde{\psi}_{t}$ is a local automorphism of $L_{V} Y$. Thus, $R_{g *} \xi_{L_{V} Y}(w)=\xi_{L_{V} Y}\left(R_{g}(w)\right)$. Because $i^{*} \theta$ is $G_{\mathcal{A}^{-}}$-tensorial, it now follows that $\left.\left.R_{g}^{*}\left(\xi_{L_{V} Y}\right\lrcorner i^{*} \theta\right)(w)=g^{-1} \cdot\left(\xi_{L_{V} Y}\right\lrcorner i^{*} \theta\right)(w)$. So $\left.\xi_{L_{V} Y}\right\lrcorner i^{*} \theta \in T_{V}^{1}\left(L_{V} Y\right)$. The proof that $J$ is $\mathrm{Ad}^{*}$ equivariant is exactly analogous to [1, Thm. 4.2.10]. By Proposition 3.1 any element of $T_{V}^{1}\left(L_{V} Y\right)$ can be identified with some $\xi$ in $\mathcal{X}_{\text {Proj }} Y$ and thus $\hat{J}(\xi)$. 
Let $\xi, \zeta \in \mathcal{X}_{\text {Proj }} Y$ and let $J$ be the momentum mapping induced by $\hat{\Psi}$. Define an $\mathbb{R}^{n+k}$ valued Poisson bracket by

$$
\{\hat{J}(\xi), \hat{J}(\zeta)\}^{\mu}=-d i^{*} \theta^{\mu}\left(\xi_{L_{V} Y}, \zeta_{L_{V} Y}\right), \quad \mu=1,2, \ldots, n+k .
$$

By Proposition 3.2, identities (2.8), and the fact that $\pi_{* Y L_{V} Y}\left[\xi_{L_{V} Y}, \zeta_{L_{V} Y}\right]=\left[\xi_{Y}, \zeta_{Y}\right]$,

$$
\{\hat{J}(\xi), \hat{J}(\zeta)\}=\hat{J}([\xi, \zeta]) .
$$

Observe that (3.7) has no additional term, in contrast to (2.9).

\section{Generalizing multisymplectic momentum mappings}

Define a linear left action of $G_{\mathcal{A}}$ on the vector space $\mathbb{R}^{n \times k} \times \mathbb{R}$ as follows.

$$
(N, K, A) \cdot(B, \lambda):=\operatorname{det}\left(N^{-1}\right)\left(N B K^{-1}, \lambda-\operatorname{tr}\left(B K^{-1} A\right)\right)
$$

The associated vector bundle $L_{V} Y \times_{G_{\mathcal{A}}}\left(\mathbb{R}^{n \times k} \times \mathbb{R}\right)$ is constructed using the actions in (3.1) and (4.1). For a point $\left(y,\left\{e_{i}, \epsilon_{A}\right\}\right) \in L_{V} Y$, define

$$
\left.\omega(e):=e^{1} \wedge e^{2} \wedge \cdots \wedge e^{n} \quad \text { and } \quad \omega(e)_{i}:=e_{i}\right\lrcorner \omega(e),
$$

where $\left\{e^{i}\right\}$ is the dual basis of $\left\{e_{i}\right\}$. From (3.2), if $\left(y,\left\{e_{i}^{\prime}, \epsilon_{A}^{\prime}\right\}\right)=\left(y,\left\{e_{i}, \epsilon_{A}\right\}\right) \cdot(N, K, A)$ then $\omega\left(e^{\prime}\right)=\operatorname{det}\left(N^{-1}\right) \omega(e)$ where $e=\left\{e_{i}\right\}$ and $e^{\prime}=\left\{e_{i}^{\prime}\right\}$.

Theorem 4.1 The map

$$
\begin{aligned}
\hat{\rho}: L_{V} Y \times_{G_{\mathcal{A}}}\left(\mathbb{R}^{n \times k} \times \mathbb{R}\right) & \rightarrow \wedge^{n} Y \\
{\left[\left(y,\left\{e_{i}, \epsilon_{A}\right\}\right),(B, \lambda)\right] } & \mapsto\left(y, B_{A}^{i} \epsilon^{A} \wedge \omega(e)_{i}+\lambda \omega(e)\right)
\end{aligned}
$$

is a vector bundle monomorphism over $Y$. The range of $\hat{\rho}$ is $Z$.

The proof of the theorem appears in [10]. Thus, the multisymplectic phase space $Z$ is a vector bundle over $Y$ associated to $L_{V} Y$. Also, the jet bundle $J Y$ is associated to $L_{V} Y$, 10, 12], but this construct is not necessary for us to proceed here. From Theorem 4.1, the relationships between local coordinates $\left\{x^{i}, y^{A}, p_{B}^{j}, p\right\}$ on $Z$ and $\left\{x^{i}, y^{A}, \pi_{k}^{j}, \pi_{C}^{B}, \pi_{l}^{D}\right\}$ on $L_{V} Y$ are

$$
p_{B}^{j}=\operatorname{det}\left(\pi_{b}^{a}\right) B_{A}^{i} \pi_{B}^{A}\left(\pi^{-1}\right)_{i}^{j} \quad \text { and } \quad p=\operatorname{det}\left(\pi_{b}^{a}\right)\left(B_{A}^{i} \pi_{k}^{A}\left(\pi^{-1}\right)_{i}^{k}+\lambda\right) .
$$

Let $B \in \mathbb{R}^{n \times k}$ and $\lambda \in \mathbb{R}$. Define the map

$$
\phi_{(B, \lambda)}: L_{V} Y \rightarrow Z: w \mapsto \hat{\rho}[w,(B, \lambda)]
$$

The map $\phi_{(B, \lambda)}$ preserves fibers over $Y$, and its range is a subbundle of $Z$ with standard fiber the $G_{\mathcal{A}}$-orbit of $(B, \lambda)$. The $G_{\mathcal{A}}$-orbits for all nonzero $B \in \mathbb{R}^{n \times k}$ are classified by the rank of $B$. 
Using Proposition 3.1 we may establish that for $\xi \in \mathcal{X}_{\text {Proj }} Y$,

$$
\phi_{(B, \lambda)_{*}} \xi_{L_{V} Y}=\xi_{Z}
$$

This is analogous to [16, Thm. 5.2] and is easily verified by local coordinate calculations.

In order to derive the multisymplectic potential $\Theta$ on $Z$ from the $(n+k)$-symplectic potential $i^{*} \theta$ on $L_{V} Y$, we must make some preliminary remarks. Let $\left\{R_{\mu}\right\}, \mu=1, \ldots, n+k$, be the standard basis of $\mathbb{R}^{n+k}$ and let $\left\{R^{\mu}\right\}$ be the corresponding dual basis. Define the $\wedge^{r+s} \mathbb{R}^{n+k}$-valued $(p+q)$-form $R_{\mu_{1} \cdots \mu_{m}}:=R_{\mu_{1}} \wedge \cdots \wedge R_{\mu_{m}} \in \wedge^{m} \mathbb{R}^{n+k}$ and $R^{\mu_{1} \cdots \mu_{m}}:=$ $R^{\mu_{1}} \wedge \cdots \wedge R^{\mu_{m}} \in \wedge^{m} \mathbb{R}^{n+k^{*}}$. Let $\alpha$ be a $\wedge^{r} \mathbb{R}^{n+k}$-valued $p$-form and let $\beta$ be a $\wedge^{s} \mathbb{R}^{n+k_{-}}$ valued $q$-form on a manifold. Then $\alpha=\alpha^{\mu_{1} \cdots \mu_{r}} \otimes R_{\mu_{1} \cdots \mu_{r}}$ and $\beta=\beta^{\mu_{1} \cdots \mu_{s}} \otimes R_{\mu_{1} \cdots \mu_{s}}$. Define

$$
\alpha \wedge \beta:=\left(\alpha^{\mu_{1} \cdots \mu_{r}} \wedge \beta^{\nu_{1} \cdots \nu_{s}}\right) \otimes R_{\mu_{1} \cdots \mu_{r} \nu_{1} \cdots \nu_{s}} .
$$

Let $\left(y,\left\{e_{i}, \epsilon_{A}\right\}\right) \in L_{V} Y$. From (4.2),

$$
\omega(e):=\frac{1}{n !} \epsilon_{i_{1} \cdots i_{n}} e^{i_{1}} \wedge \cdots \wedge e^{i_{n}} \quad \text { and } \quad \omega(e)_{j}:=\frac{1}{(n-1) !} \epsilon_{j i_{1} \cdots i_{n-1}} e^{i_{1}} \wedge \cdots \wedge e^{i_{n-1}}
$$

where $\epsilon_{i_{1} \ldots i_{n}}$ is the sign of the permutation $(1, \ldots, n) \mapsto\left(i_{1}, \ldots, i_{n}\right)$.

Theorem 4.2 For $n \geq 2$, the $\wedge^{n} \mathbb{R}^{n+k}$-valued $n$-form $i^{*} \wedge^{n} \theta$ on $L_{V} Y$ can be related to the canonical $n$-form $\Theta$ on $Z$ by

$$
\left\langle\wedge^{n} i^{*} \theta, \boldsymbol{V}(B, \lambda)\right\rangle=\phi_{(B, \lambda)}^{*} \Theta
$$

where the map $\boldsymbol{V}: \mathbb{R}^{n \times k} \times \mathbb{R} \rightarrow \wedge^{n} \mathbb{R}^{n+k^{*}}$ has components

$$
\begin{aligned}
V_{i_{1} \ldots i_{n}}(B, \lambda) & =\frac{1}{n !} \lambda \epsilon_{i_{1} \ldots i_{n}}, \\
V_{A i_{1} \ldots i_{n-1}}(B, \lambda) & =\frac{1}{n !} B_{A}^{j} \epsilon_{j i_{1} \ldots i_{n-1}} \\
\text { and } \quad V_{A_{1} \ldots A_{l} i_{1} \ldots i_{n-l}}(B, \lambda) & =0 \forall l \geq 2 .
\end{aligned}
$$

The theorem is proven in [10]. Equation (4.5) holds for $n=1$ if $V_{i}(B, \lambda)=\lambda$ and $V_{A}(B, \lambda)=$ $B_{A}$.

Lemma 4.3 Let $\bar{\Psi}$ be the lift to $Z$ of the representation $\Psi$ of Aut $Y$ on $Y$. Let $B \in \mathbb{R}^{n \times k}$ and $\lambda \in \mathbb{R}$. Then, using the identification $L_{V} Y \times_{G_{\mathcal{A}}}\left(\mathbb{R}^{n \times k} \times \mathbb{R}\right) \simeq Z$ from Theorem 4.1.

$$
\bar{\Psi}\left(\eta_{Y},\left[\left(y,\left\{e_{i}, \epsilon_{A}\right\}\right),(B, \lambda)\right]\right) \simeq\left[\tilde{\Psi}\left(\eta_{Y},\left(y,\left\{e_{i}, \epsilon_{A}\right\}\right)\right),(B, \lambda)\right] .
$$

As a consequence, $\phi_{(B, \lambda)} \circ \tilde{\Psi}_{f}(w)=\hat{\Psi}_{f} \circ \phi_{(B, \lambda)}(w)$.

Proof Using the action in (4.1) and Theorem 4.1,

$$
\begin{aligned}
{\left[\tilde{\Psi}\left(\eta_{Y},\left(y,\left\{e_{i}, \epsilon_{A}\right\}\right)\right),(B, \lambda)\right] } & \simeq\left(\eta_{Y}(y), B_{A}^{i}\left(\eta_{Y *} \epsilon\right)^{* A} \wedge \omega\left(\eta_{Y *} e\right)_{i}+\lambda \omega\left(\eta_{Y *} e\right)\right) \\
& =\left(\eta_{Y}(y), \eta_{Y}^{-1 *}\left(B_{A}^{i} \epsilon^{A} \wedge \omega(e)_{i}+\lambda \omega(e)\right)\right) \\
& \simeq \bar{\Psi}\left(\eta_{Y},\left[\left(y,\left\{e_{i}, \epsilon_{A}\right\}\right),(B, \lambda)\right]\right) .
\end{aligned}
$$


By choosing representatives of the equivalence classes, we see that $\phi_{(B, \lambda)} \circ \tilde{\Psi}_{f}(w)=\hat{\Psi}_{f} \circ$ $\phi_{(B, \lambda)}(w)$.

Theorem 4.4 Let $w \in L_{V} Y$ and $(B, \lambda) \in \mathbb{R}^{n \times k} \times \mathbb{R}$.

$$
\phi_{(B, \lambda)}^{*}\left(\hat{J}_{Z}(\xi)\right)=\left\langle\hat{J}_{L_{V} Y}(\xi) \wedge\left(\wedge^{n-1} i^{*} \theta\right), n \boldsymbol{V}(B, \lambda)\right\rangle
$$

where $\boldsymbol{V}(B, \lambda) \in \wedge^{n} \mathbb{R}^{(n+k) *}$ is defined in Theorem 4.9 .

Proof Let $w=\left(y,\left\{e_{i}, \epsilon_{A}\right\}\right) \in L_{V} Y$, and let $\psi_{t}$ be a local one-parameter group of $X$. Then $\tilde{\psi}_{t}$ is a flow on $L_{V} Y$ and $\hat{\psi}_{t}$ is a flow on $Z$. It follows from Lemma 4.3 and equation (4.4) that $\phi_{(B, \lambda)} \circ \tilde{\psi}_{t}(w)=\hat{\psi}_{t} \circ \phi_{(B, \lambda)}(w)$ and thus $\phi_{(B, \lambda)_{*}} \xi_{L_{V} Y}(w)=\xi_{Z}\left(\phi_{(B, \lambda)}(w)\right)$. Using equation (2.3) and Theorem 4.2,

$$
\begin{aligned}
\left\langle\hat{J}_{L_{V} Y}(\xi) \wedge\left(\wedge^{n-1} i^{*} \theta\right), n \boldsymbol{V}(B, \lambda)\right\rangle & \left.=\left\langle\left(\xi_{L_{V} Y}\right\lrcorner \wedge^{n} i^{*} \theta\right), \boldsymbol{V}(B, \lambda)\right\rangle \\
& \left.=\xi_{L_{V} Y}\right\lrcorner \phi_{(B, \lambda)}{ }^{*} \Theta \\
& \left.=\phi_{(B, \lambda)}{ }^{*}\left(\xi_{Z}\right\lrcorner \Theta\right) \\
& =\phi_{(B, \lambda)}{ }^{*} \hat{J}_{Z}(\xi) .
\end{aligned}
$$

The last theorem motivates us to represent $T_{V}^{1}\left(L_{V} Y\right)$ in the space of $\wedge^{m+1} \mathbb{R}^{n+k}$-valued $m$-forms by

$$
\hat{J}(\xi) \mapsto \hat{J}(\xi) \wedge\left(\wedge^{m} i^{*} \theta\right)
$$

for $0 \leq m \leq n+k$. By induction,

$$
\left.\xi_{L_{V} Y}\right\lrcorner \wedge^{m} i^{*} \theta=m \hat{J}(\xi) \wedge\left(\wedge^{m-1} i^{*} \theta\right) .
$$

Because for each $0 \leq m \leq n+k-2$ the form $d\left(\wedge^{m+1} i^{*} \theta\right)$ is closed and nondegenerate, it is a candidate for a higher degree generalization of the $(n+k)$-symplectic form $i^{*} d \theta$. Using (4.7) and

$$
d\left(\wedge^{m} \theta\right)=m d \theta \wedge\left(\wedge^{m-1} \theta\right)
$$

it follows that

$$
\left.d\left(\hat{J}(\xi) \wedge\left(\wedge^{m} i^{*} \theta\right)\right)=-\xi_{L_{V} Y}\right\lrcorner\left(i^{*} d \theta \wedge\left(\wedge^{m} i^{*} \theta\right)\right) .
$$

For $0 \leq m \leq n+k$ define a bracket on the image of representation (4.6) by

$$
\left.\left.\left\{\hat{J}(\xi) \wedge\left(\wedge^{m} i^{*} \theta\right), \hat{J}(\zeta) \wedge\left(\wedge^{m} i^{*} \theta\right)\right\}:=\xi_{L_{V} Y}\right\lrcorner \zeta_{L_{V} Y}\right\lrcorner\left(i^{*} d \theta \wedge\left(\wedge^{m} i^{*} \theta\right)\right) .
$$

If $m=0$ then this becomes the bracket on $T_{V}^{1}\left(L_{V} Y\right)$ given by (3.6). This sign convention for the bracket is consistent with [6] and is the negative of that of [15]. Computation on the forms yields, for $0 \leq m \leq n+k$,

$$
\left\{\hat{f} \wedge\left(\wedge^{m} i^{*} \theta\right), \hat{g} \wedge\left(\wedge^{m} i^{*} \theta\right)\right\}=\{\hat{f}, \hat{g}\} \wedge\left(\wedge^{m} i^{*} \theta\right)+m d\left(\hat{f} \wedge \hat{g} \wedge\left(\wedge^{m-1} i^{*} \theta\right)\right) .
$$


Equation (4.8) is of particular interest when $m=n-1, \hat{f}=\hat{J}(\xi)$, and $\hat{g}=\hat{J}(\zeta)$. In this case we have reproduced on $L_{V} Y$ the exact analogue of the problem in (2.9) that $T^{1}(Z)$ is not closed under its "Poisson" bracket. We could extend the algebra of momentum observables on $L_{V} Y$ under representation (4.6) to include closed $\mathbb{R}^{n+k}$-valued $(n-1)$-forms but its representation into $H V^{1}\left(L_{V} Y\right)$ would have a kernel larger than that of the representation from $T_{V}^{1}\left(L_{V} Y\right)$ into the same space of vector fields. The space $T_{V}^{1}\left(L_{V} Y\right)$ of $\mathbb{R}^{n+k}$-valued Hamiltonian tensorial observables already possesses a well-defined Lie algebra under (3.6), Proposition 3.1 shows that $T_{V}^{1}\left(L_{V} Y\right)$ and $T^{1}(Z)$ are in bijective correspondence, and equation (4.4) relates the corresponding spaces of Hamiltonian vector fields. Thus the $(n+k)$-symplectic geometry of $L_{V} Y$ not only generates the multisymplectic geometry of $Z$ for classical fields but also possesses an algebraic structure that $Z$ lacks.

\section{Conserved quantities}

Let $S_{\mu \nu}$ denote $R_{\mu} \otimes_{s} R_{\nu}$, where $\otimes_{s}$ is the symmetric tensor product.

\section{Definitions}

- Let $S T^{2}\left(L_{V} Y\right)$ denote the vector space of tensorial $\mathbb{R}^{n+k} \otimes_{s} \mathbb{R}^{n+k}$-valued functions on $L_{V} Y$.

- Let $H F^{2}\left(L_{V} Y\right)$ denote the vector space of $\mathbb{R}^{n+k} \otimes_{s} \mathbb{R}^{n+k}$-valued functions $\hat{g}=\hat{g}^{\mu \nu} S_{\mu \nu}$ on $L_{V} Y$ that satisfy the symmetrized $(n+k)$-symplectic equation

$$
\left.d \hat{g}^{\mu \nu}=-2 X^{(\mu}\right\lrcorner i^{*} d \theta^{\nu)}
$$

where $X^{\mu} R_{\mu}$ is an $\mathbb{R}^{n+k}$-valued vector field and parentheses denote symmetrization.

- Let $H V^{2}\left(L_{V} Y\right)$ denote the set of $\mathbb{R}^{n+k}$-valued vector fields $X_{\hat{g}}^{\mu} R_{\mu}$ satisfying equation (5.1) for some $\hat{g}^{\mu \nu} \in H F^{2}\left(L_{V} Y\right)$. We shall call such vector fields Hamiltonian.

- Let $S T_{V}^{2}\left(L_{V} Y\right):=S T^{2}\left(L_{V} Y\right) \cap H F^{2}\left(L_{V} Y\right)$.

Tensorial properties require that $\hat{g} \in S T^{2}\left(L_{V} Y\right)$ appear in local coordinates as

$$
\begin{aligned}
\hat{g}(u)= & g^{i j} \pi_{i}^{k} \pi_{j}^{l} S_{k l}+g^{i j} \pi_{i}^{A} \pi_{j}^{l} S_{l A}+g^{i A} \pi_{i}^{l} \pi_{A}^{B} S_{l B} \\
& +g^{i j} \pi_{i}^{A} \pi_{j}^{B} S_{A B}+g^{i C} \pi_{i}^{A} \pi_{C}^{B} S_{A B}+g^{C D} \pi_{C}^{A} \pi_{D}^{B} S_{A B},
\end{aligned}
$$

where the component functions $g^{i j}, g^{i A}$, and $g^{A B}$ are functions of $x^{k}$ and $y^{A}$. If $\hat{g} \in$ $S T_{V}^{2}\left(L_{V} Y\right)$ then the only additional restriction we have is that $g^{i j}=g^{i j}\left(x^{k}\right)$. So $S T_{V}^{2}\left(L_{V} Y\right)$ is in bijective correspondence with the space of projectable symmetric tensors of degree 2 on $Y$. This is in exact analogy to Proposition 3.1.

As in the theory on $L M$ [15], symmetrization in equation (5.1) means that the $\mathbb{R}^{n+k}$ valued Hamiltonian vector fields $X_{\hat{g}}^{\mu} R_{\mu}$ are not uniquely determined by $\hat{g} \in S T_{V}^{2}\left(L_{V} Y\right)$. Rather, they are determined locally up to the addition of vector fields $Y^{\mu} R_{\mu}$ which satisfy

$$
\left.Y^{(\mu}\right\lrcorner i^{*} d \theta^{\nu)}=0 \text {. }
$$


Thus each $Y^{\mu}$ must be a vertical vector field. For a given $\hat{g} \in S T_{V}^{2}\left(L_{V} Y\right)$, two $\mathbb{R}^{n+k}$-valued Hamiltonian vector fields are in the same equivalence class $\llbracket X_{\hat{f}} \rrbracket^{\mu} R_{\mu}$ if their difference $Y^{\mu}$ satisfies (5.3). Obtaining vector fields only up to equivalence does not affect the basic algebraic structures of the $(n+k)$-symplectic geometry on $L_{V} Y$. For example, if $\hat{f} \in$ $T_{V}^{1}\left(L_{V} Y\right)$ and $\hat{g} \in S T_{V}^{2}\left(L_{V} Y\right)$, define a Poisson bracket by

$$
\{\hat{f}, \hat{g}\}:=-X_{\hat{f}}\left(\hat{g}^{\mu \nu}\right) S_{\mu \nu}
$$

or use any representative of the equivalence class $\llbracket X_{\hat{g}} \rrbracket^{\mu}$ to define a Poisson bracket by

$$
\{\hat{g}, \hat{f}\}=-2 X_{\hat{g}}^{(\mu}\left(\hat{f}^{\nu)}\right) S_{\mu \nu} .
$$

The two are related by $\{\hat{g}, \hat{f}\}=-\{\hat{f}, \hat{g}\}$. The algebra may be extended to higher degree observables and tensor-valued Hamiltonian vector fields, generating an algebra corresponding to the symmetric differential concomitants of Schouten [19] and Nijenhuis [14]. See [15, 16, 17] for details on how this is done on $L M$. The proofs easily extend to $L_{V} Y$.

Lemma 5.1 Let $\hat{f}=\hat{f}^{\mu} R_{\mu} \in T_{V}^{1}\left(L_{V} Y\right)$ and let $X_{\hat{f}}$ be its corresponding Hamiltonian vector field. Let $\hat{g} \in S T_{V}^{2}\left(L_{V} Y\right)$ and let $X_{\hat{g}}$ be a representative of its equivalence class $\llbracket X_{\hat{g}} \rrbracket$ of corresponding $\mathbb{R}^{n+k}$-valued Hamiltonian vector fields. If $\{\hat{g}, \hat{f}\}=0$, then for each $\mu=$ $1,2, \ldots, n+k, \hat{f}^{\mu}$ is constant on the orbits of each $X_{\hat{g}}{ }^{\mu} \in \llbracket X_{\hat{g}} \rrbracket^{\mu}$.

Proof [16] Let $F_{t}^{\mu}$ be the flow of $X_{\hat{g}}^{\mu} \in \llbracket X_{\hat{g}} \rrbracket^{\mu}$. Then

$$
\frac{d}{d t}\left(\hat{f}^{\mu} \circ F_{t}^{\mu}\right)=\left(F_{t}^{\mu}\right)^{*}\left(\mathbf{L}_{X_{\hat{g}}^{\mu}}\left(\hat{f}^{\mu}\right)\right)=\left(F_{t}^{\mu}\right)^{*}\left(X_{\hat{g}}^{\mu}\left(\hat{f}^{\mu}\right)\right)=-\frac{1}{2}\left(F_{t}^{\mu}\right)^{*}\left(\{\hat{g}, \hat{f}\}^{\mu \mu}\right) .
$$

So if $\{\hat{g}, \hat{f}\}=0$ then $\frac{d}{d t}\left(\hat{f}^{\mu} \circ F_{t}^{\mu}\right)=0$. This holds regardless of the choice of representative of $\llbracket X_{\hat{g}} \rrbracket^{\mu}$.

Theorem 5.2 Let $\Phi$ be an $(n+k)$-symplectic action of a subgroup $H$ of Aut $Y$ upon $L_{V} Y$, and let $J$ be the corresponding momentum mapping. Suppose that $\hat{g} \in S T_{V}^{2}\left(L_{V} Y\right)$ is invariant under the action. Then $J$ provides $n+k$ integrals of $\hat{g}$ in the sense that

$$
J^{\mu}\left(F_{t}^{\mu}(u)\right)=J^{\mu}(u),
$$

where $F_{t}^{\mu}$ is the flow of any representative of $\llbracket X_{\hat{g}} \rrbracket^{\mu}, \mu=1,2, \ldots, n+k$.

Proof The proof follows [16, Appendix 1] and [1, Theorem 4.2.2]. Let $\mathfrak{h}$ be the Lie algebra of $H$. From our remarks in Section 2, if $H \subset$ Aut $Y$ then $\mathfrak{h} \subset \mathcal{X}_{\text {Proj }} Y$. For each $\xi$ in $\mathfrak{h}$, invariance of $\hat{g}$ under $\Phi$ implies

$$
\hat{g}\left(\Phi_{\exp (t \xi)}(u)\right)=\hat{g}(u) .
$$

Differentiating (5.4) at $t=0$ gives us $d \hat{g}\left(\xi_{L_{V} Y}\right)=0$, which implies that

$$
\mathbf{L}_{\xi_{L_{V} Y}}(\hat{g})=0 .
$$


By Lemma 3.3 if $\xi \in \mathcal{X}_{\text {Proj }} Y$, then $\hat{J}(\xi) \in T_{V}^{1}\left(L_{V} Y\right)$. This and (5.5) imply that

$$
\{\hat{J}(\xi), \hat{g}\}=0 .
$$

Hence, by Lemma 5.1,

$$
(\hat{J}(\xi))^{\mu}\left(F_{t}^{\mu}(u)\right)=(\hat{J}(\xi))^{\mu}(u)
$$

Thus, by the definition of a momentum mapping on $L_{V} Y$,

$$
J^{\mu}\left(F_{t}^{\mu}(u)\right)=J^{\mu}(u)
$$

\section{Examples}

\subsection{Linear momentum in field theories}

Following examples in [1] and [16], we express linear momentum in the language of momentum mappings on $L_{V} Y$ and on $Z$. Let $X=\mathbb{R}^{n}, Y=\mathbb{R}^{n+k}$, and $\mathcal{G}=\left(\mathbb{R}^{n+k},+\right.$ ). (So $\mathfrak{g}$ is the abelian Lie algebra $\mathbb{R}^{n+k}$.) Define an action of $\mathcal{G}$ on $Y$ by

$$
\Psi: \mathcal{G} \times Y \rightarrow Y:(g, y) \mapsto \Psi_{g}(y):=g+y .
$$

Define

$$
\underline{\Psi}: \mathcal{G} \times X \rightarrow X:(g, x) \mapsto \underline{\Psi}_{g}(x):=\operatorname{Pr}_{1}(g)+x,
$$

where $\operatorname{Pr}_{1}: \mathbb{R}^{n+k} \rightarrow \mathbb{R}^{n}$ is projection onto the first factor. Then $\Psi_{g}$ and $\underline{\Psi}_{g}$ are bijective and $\underline{\Psi}_{g} \circ \pi=\pi \circ \Psi_{g}$. Thus $g \mapsto \Psi_{g}$ is a representation of $\mathcal{G}$ in $\operatorname{Aut}(Y)$. Now we may compute the generators of the action. First, define a lift $\tilde{\Psi}$ of the representation $\Psi$ to $L_{V} Y$ by

$$
\begin{aligned}
\tilde{\Psi}\left(g,\left(y,\left\{e_{i}, \epsilon_{A}\right\}\right)\right) & =\left(\Psi_{g}(y),\left\{\Psi_{g *} e_{i}, \Psi_{g *} \epsilon_{A}\right\}\right) \\
& =\left(y+g,\left\{e_{i}, \epsilon_{A}\right\}\right) .
\end{aligned}
$$

Let $\xi \in \mathfrak{g}$. The infinitesimal generator on the action on $L_{V} Y$ is

$$
\xi_{L_{V} Y}\left(y,\left\{e_{i}, \epsilon_{A}\right\}\right)=\left.\frac{d}{d t}\left(y+\exp t X,\left\{e_{i}, \epsilon_{A}\right\}\right)\right|_{t=0} .
$$

If $\xi_{L_{V} Y}=\xi^{i} \hat{r}_{i}+\xi^{A} \hat{s}_{A}$ then we may write equation (6.4) locally on $L_{V} Y$ as

$$
\xi_{L_{V} Y}=\xi^{i} \frac{\partial}{\partial x^{i}}+\xi^{A} \frac{\partial}{\partial y^{A}} .
$$

From equations (3.3) and (6.5), the corresponding momentum mapping is

$$
\left.\hat{J}_{L_{V} Y}(\xi)=\xi_{L_{V} Y}\right\lrcorner i^{*} \theta=\xi^{i} \pi_{i}^{j} \hat{r}_{j}+\xi^{A} \pi_{A}^{B} \hat{s}_{B}+\xi^{i} \pi_{i}^{B} \hat{s}_{B}
$$

or

$$
J_{L_{V} Y}=\pi_{i}^{j} \hat{r}^{i} \otimes \hat{r}_{j}+\pi_{B}^{A} \hat{s}^{B} \otimes \hat{s}_{A}+\pi_{i}^{B} \hat{r}^{i} \otimes \hat{s}_{B}
$$


The $\mathbb{R}^{n+k}$-valued function $\hat{J}_{L_{V} Y}(\xi)$ is interpreted as a momentum frame 16 .

Now consider linear momentum on $Z$, using the same $\mathcal{G}$ and $Y$ as before. The lift of $\Psi$ to $Z$ is given by

$$
\hat{\Psi}_{g}(z)=\Psi_{g}^{-1 *} z
$$

As with the case on $L_{V} Y$, in local coordinates,

$$
\xi_{Z}(z)=\xi^{i} \frac{\partial}{\partial x^{i}}+\xi^{A} \frac{\partial}{\partial y^{A}} .
$$

On $\mathrm{Z}$ we may write

$$
\left.\hat{J}_{Z}(\xi)=\xi_{Z}\right\lrcorner \Theta
$$

or locally,

$$
\hat{J}_{Z}(\xi)=\xi^{A} p_{A}^{i}\left(d^{n-1} x\right)_{i}+\xi^{i} p_{A}^{j} d y^{A} \wedge\left(d^{n-2} x\right)_{i j}+\xi^{i} p\left(d^{n-1} x\right)_{i},
$$

which has the interpretation of the linear momentum of a field and is consistent with [6, p. 45]. To verify Theorem 4.4, namely that $\left\langle\hat{J}_{L_{V} Y}(\xi) \wedge\left(\wedge^{n-1} i^{*} \theta\right), \boldsymbol{V}\right\rangle=\phi_{(B, \lambda)}{ }^{*}\left(\hat{J}_{Z}(\xi)\right)$, we may perform a local coordinate calculation comparing (6.7) with (6.6).

\subsection{Angular momentum in field theories}

Motivated by examples on the cotangent bundle [1] and on the linear frame bundle [16], we express angular momentum in the language of momentum mappings on $L_{V} Y$ and on $Z$. Let $X=\mathbb{R}^{n}$ and $Y=\mathbb{R}^{n+k}$ and let $\left\{x^{i}, y^{A}\right\}$ be adapted coordinates on $Y$. Let $\eta$ be a constant nondegenerate metric on $\mathbb{R}^{n}$ and let $\iota$ be a constant nondegenerate metric on $\mathbb{R}^{k}$. (For example, to model the space of 4-vector electromagnetic potentials over Minkowski spacetime, both $\eta$ and $\iota$ could be the Lorentz metric on $\mathbb{R}^{4}$.) Construct the Kaluza-Klein metric $G$ on $Y$ using an Ehresmann connection $\gamma: T Y \rightarrow V(T Y)$. The Kaluza-Klein metric is the fiberwise bilinear map $\mathrm{G}$ on $T Y$ given by

$$
G(v, w)=\pi_{X Y}^{*} \eta(v, w)+\iota(\gamma(v), \gamma(w)) .
$$

If we use the trivial Ehresmann connection $\gamma: \mathbb{R}^{n+k} \rightarrow \mathbb{R}^{n}: v^{i} \hat{r}_{i}+v^{A} \hat{s}_{A} \mapsto v^{A}$, then $G$ is itself constant and nondegenerate, $G \mid \gamma(T X)=\eta$, and $G \mid V(T Y)=\iota$. In adapted coordinates,

$$
G(v, w)=\eta_{i j} v^{i} w^{j}+\iota_{A B} v^{A} w^{B} .
$$

Define a left action $\Phi:\left(O_{\eta}(n) \times O_{\iota}(k)\right) \times Y \rightarrow Y$ by left matrix multiplication on column vectors in $\mathbb{R}^{n+k}$, and $\Phi_{g}(y):=\Phi(g, y)$. Define a left action

$$
\underline{\Phi}:\left(O_{\eta}(n) \times O_{\iota}(k)\right) \times X \rightarrow X
$$

by $\underline{\Phi}(g, x)=\pi \circ \Phi(g \hat{x})=: \underline{\Phi}_{g}(x)$ where $\mathbb{R}^{n} \hookrightarrow \mathbb{R}^{n+k}: x \mapsto \hat{x}$ is inclusion. Observe that $\Phi_{g}$ and $\underline{\Phi}_{g}$ are bijections and that $\underline{\Phi}_{g} \circ \pi=\pi \circ \Phi_{g}$. Thus $g \mapsto \Phi_{g}$ is a representation of $O_{\eta}(n) \times O_{\iota}(k)$ in $\operatorname{Aut}(Y)$. 
Remark The larger group $O_{G}(n+k)$ does not possess a nontrivial left action on $X=\mathbb{R}^{n}$. Under left multiplication of $G L(n+k)$ on $\mathbb{R}^{n+k}, g \in G L(n+k)$ leaves $\mathbb{R}^{n}$ invariant if and only if $g \in G_{A}$. Thus, we use $O_{\eta}(n) \times O_{\iota}(k)=G_{A} \cap O_{G}(n+k)$.

To compute the infinitesimal generators, let $\xi=\xi_{\nu}^{\mu} E_{\mu}^{\nu} \in \mathfrak{o}_{\eta}(n) \times \mathfrak{o}_{\iota}(k)$, where $\left\{E_{\mu}^{\nu}\right\}$ is the standard basis for the Lie algebra $\mathfrak{g l}(n+k)$ and the subset $\left\{E_{j}^{i}, E_{B}^{A}\right\}$ is a basis for $\mathfrak{o}_{\eta}(n) \times \mathfrak{o}_{\iota}(k)$. Then the infinitesimal generator of the action of $\xi$ on $Y$ is

$$
\xi_{Y}(y)=\xi_{j}^{i} x^{j} \frac{\partial}{\partial x^{i}}+\xi_{B}^{A} y^{B} \frac{\partial}{\partial y^{A}}
$$

Note that $\pi_{X Y *}\left(\xi_{Y}(y)\right)=\xi_{j}^{i} x^{j} \frac{\partial}{\partial x^{i}}=\xi_{X}\left(\pi_{X Y *}(y)\right)$. Observe that $\xi_{Y}$ is a Killing vector field of $G$. Indeed it is easy to verify that

$$
G_{\mu \nu}\left(\xi_{Y}\right)^{\nu}{ }_{\kappa}+G_{\kappa \nu}\left(\xi_{Y}\right)^{\nu}, \mu=0 .
$$

If $\kappa=C$ and $\mu=D$, then the left side of (6.8) simplifies to $\iota_{D A} \xi_{C}^{A}+\xi_{D}^{A} \iota_{A C}$, which vanishes because $\left(\xi_{B}^{A}\right) \in \mathcal{O}_{\iota}(k)$. If $\kappa=k$ and $\mu=l$, then the left side of (6.8) becomes $\eta_{l i} \xi_{k}^{i}+\xi_{l}^{i} \eta_{i k}$, which vanishes because $\left(\xi_{j}^{i}\right) \in \mathcal{O} \eta(n)$. If $\kappa=l$ and $\mu=C$ or vice-versa, the left side of (6.8) is identically zero since $G_{i A}=0$.

Let $u \in L_{V} Y$ and $\xi \in \mathfrak{o}_{\eta}(n) \times \mathfrak{o}_{\iota}(k)$. The momentum mapping on $L_{V} Y$ associated with the action defined above is

$$
\hat{J}_{L_{V} Y}(\xi)(u)=\xi_{k}^{j} x^{k} \pi_{j}^{i} \hat{r}_{i}+\left(\xi_{k}^{j} x^{k} \pi_{j}^{A}+\xi_{C}^{B} y^{C} \pi_{B}^{A}\right) \hat{s}_{A},
$$

and if $\left(C_{\nu}^{\mu}\right)$ is the basis of $\mathfrak{g l}(n+k)^{*}$ dual to $\left(E_{\mu}^{\nu}\right)$ then

$$
\begin{aligned}
J(u) & =C_{k}^{j} x^{k} \pi_{j}^{i} \hat{r}_{i}+\left(C_{k}^{j} x^{k} \pi_{j}^{A}+C_{D}^{B} y^{D} \pi_{B}^{A}\right) \hat{s}_{A} \\
& =\eta_{k l} C^{l j} x^{k} \pi_{j}^{i} \hat{r}_{i}+\left(\eta_{k l} C^{j l} x^{k} \pi_{j}^{A}+\iota_{D E} C^{D B} y^{E} \pi_{B}^{A}\right) \hat{s}_{A} \\
& =\frac{1}{2}\left(\left(x_{i} \pi_{j}^{k}-x_{j} \pi_{i}^{k}\right) C^{j i} \hat{r}_{k}+\left(\left(x_{i} \pi_{j}^{A}-x_{j} \pi_{i}^{A}\right) C^{j i}+\left(y_{D} \pi_{B}^{A}-y_{B} \pi_{D}^{A}\right) C^{B D}\right) \hat{s}_{A}\right) .
\end{aligned}
$$

The function $\hat{G}$ corresponding to the metric $G$ is in $S T_{V}^{2}\left(L_{V} Y\right)$, and via equation (5.1), produces a system of $n+k$ Hamiltonian vector fields,

$$
\left\{\begin{array}{l}
X_{\hat{G}}^{i}=\eta^{j k} \pi_{j}^{i} \frac{\partial}{\partial x^{k}} \\
X_{\hat{G}}^{A}=\eta^{j k} \pi_{j}^{A} \frac{\partial}{\partial x^{k}}+\iota^{B C} \pi_{B}^{A} \frac{\partial}{\partial y^{C}}
\end{array}\right.
$$

Equation (6.8) implies that the $X_{\hat{G}}^{\mu}$ are tangent to the $O_{\eta}(n) \times O_{\iota}(k)$ subbundle of $L_{V} Y$. By Theorem 5.2, each $J^{\mu}$ is constant along $X_{\hat{G}}^{\mu}$ for the same $\mu$. We may interpret $J^{i}$ to be the $i^{\text {th }}$ component of the (extrinsic) angular momentum in parameter space (e.g., Minkowski spacetime) and $J^{A}$ to be the $i^{\text {th }}$ component of the total angular momentum (both extrinsic and field) in the field configuration space. As a result, along an integral curve (a solution of Hamilton's equations for the metric Hamiltonian), the $X_{\hat{G}}^{i}$ gives the equations $\dot{x}^{k}=\eta^{k j} \pi_{j}^{i}$ and $\dot{y}^{A}=\dot{\pi}_{\nu}^{\mu}=0$. Integrating with an initial condition $u_{0}=\left(y_{0},\left\{e_{i}, \epsilon_{A}\right\}_{0}\right)$, we obtain in 
local coordinates the flow $F_{t}^{i}=\left(x^{k}+t \eta^{k j} \pi_{j}^{i}, y^{A}, \pi_{l}^{m}, \pi_{n}^{B}, \pi_{D}^{C}\right)$, which is the same as the flow of $X_{\hat{g}}^{i}$ on $X$. In fact,

$$
\begin{aligned}
J^{i} \circ F_{t}^{i} & =\pi_{j}^{i}\left(x^{k}+t \eta^{k l} \pi_{l}^{i}\right) C_{k}^{j} \quad(\text { no sum on } i) \\
& =\pi_{j}^{i} x^{k} C_{k}^{j}+t \pi_{j}^{i} \pi_{l}^{i} C^{j l} \\
& =\pi_{j}^{i} x^{k} C_{k}^{j} \\
& =g_{j l} \dot{x}^{l} x^{k} C_{k}^{j} \\
& =\dot{x}^{[l} x^{k]} C_{l k} \\
& =J^{i} .
\end{aligned}
$$

From the $X_{\hat{G}}^{A}$ we obtain $\dot{x}^{i}=\eta^{i j} \pi_{j}^{A}, \dot{y}^{C}=\iota^{B C} \pi_{B}^{A}$, and $\dot{\pi}_{\nu}^{\mu}=0$. The flow for $X_{\hat{G}}^{A}$ is

$$
F_{t}^{A}=\left(x^{k}+t \eta^{k j} \pi_{j}^{A}, y^{C}+t \iota{ }^{C B} \pi_{B}^{A}, \pi_{m}^{l}, \pi_{n}^{D}, \pi_{F}^{E}\right),
$$

and

$$
\begin{aligned}
J^{A} \circ F_{t}^{A} & \left.=\pi_{i}^{A}\left(x^{k}+t \eta^{k l} \pi_{l}^{A}\right) C_{k}^{i}+\pi_{D}^{A}\left(y^{B}+t \iota^{B E} \pi_{E}^{A}\right) C_{B}^{D} \quad \text { (no sum on } A\right) \\
& =\pi_{j}^{A} x^{k} C_{k}^{j}+\pi_{B}^{A} y^{D} C_{D}^{B}+t \pi_{i}^{A} \pi_{l}^{A} C^{i l}+t \pi_{D}^{A} \pi_{B}^{A} C^{D B} \\
& =\pi_{j}^{A} x^{k} C_{k}^{j}+\pi_{B}^{A} y^{D} C_{D}^{B} \\
& =\dot{x}^{[l} x^{k]} C_{l k}+\dot{y}^{[D} y^{B]} C_{D B} \\
& =J^{A} .
\end{aligned}
$$

Thus along a trajectory (integral curve) extrinsic angular momentum is conserved (equation (6.11)) and total angular momentum is conserved (equation (6.12)).

If $n=1$ then we may model time-evolved particle mechanics (with $k$ degrees of freedom). The group action is, up to time rescaling, merely that of the orthogonal group in the $k$ spatial dimensions. Equation (6.11) reduces to $J^{i} \circ F_{t}^{i} \equiv 0$, and equation (6.12) reduces

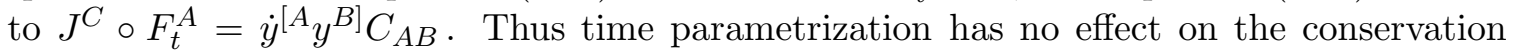
of angular momentum. If $k=1$ then we may model scalar fields over parameter space. The group action is, up to rescaling in the field, merely that of the orthogonal group in the $n$ spatial dimensions. Then equation (6.11) is left intact and equation (6.12) reduces to equation (6.11). The scalar field angular momentum comes purely from the parameter space.

\subsection{Affine symmetry in time-evolution mechanics}

Let $X=\mathbb{R}$ and let $Y=\mathbb{R} \times \mathbb{R}^{k}$, with coordinates $\left\{x^{0}=t, y^{A}\right\}$. Give $X$ the Euclidean metric, and let $\iota$ be a constant nondegenerate metric on the fiber $\mathbb{R}^{k}$. Assume the trivial connection of $Y$ over $X$ to construct the Kaluza-Klein metric $G$ on $Y$,

$$
G(v, w)=v^{0} w^{0}+\iota_{A B} v^{A} w^{B} .
$$

Construct a subgroup of $G L(k+1)$ by

$$
\mathcal{G}(k):=\left\{\left(\begin{array}{cc}
1 & 0 \\
v & K
\end{array}\right) \mid K \in O_{\iota}(k), v \in \mathbb{R}^{k}\right\} .
$$


The group $\mathcal{G}(k)$ is the semidirect product of $\mathbb{R}^{k}$ with $O_{\iota}(k)$. The left representation $\Phi$ : $\mathcal{G}(k) \times Y \rightarrow Y$ defined by left matrix multiplication covers the trivial representation of $\mathcal{G}(k)$ on $X=\mathbb{R}$, so that for all $g \in \mathcal{G}(k), \Phi_{g} \in \operatorname{Aut}(Y)$. Lift $\Phi_{g}$ to an action of $\mathcal{G}(k)$ on $L_{V} Y$. The corresponding Lie algebra $\mathfrak{g}(k)$ is the semidirect product of $\mathbb{R}^{k}$ with $\mathfrak{o}_{\iota}(k)$, and may be represented as a subalgebra of $\mathfrak{g l}(k+1)$ by

$$
\mathfrak{g}(k)=\left\{\left(\begin{array}{cc}
0 & 0 \\
v & k
\end{array}\right) \mid k \in \mathfrak{o}_{\iota}(k), v \in \mathbb{R}^{k}\right\}
$$

If we express the basis of $\mathfrak{g}(k)$ by $\left\{E_{B}^{A}, s_{C}\right\}$ where $\left\{E_{B}^{A}\right\}$ is the standard basis for $\mathfrak{o}_{\iota}(k)$ and $\left\{s_{C}\right\}$ is the standard basis for $\mathbb{R}^{k}$, For $\xi \in \mathfrak{g}(k)$, we may write $\xi=k_{B}^{A} E_{A}^{B}+v^{A} s_{A}$. In local coordinates the corresponding infinitesimal generator on $Y$ is $\xi_{Y}(y)=\left(k_{B}^{A} y^{B}+x^{0} v^{A}\right) \frac{\partial}{\partial y^{A}}$. The corresponding momentum mapping on $L_{V} Y$ is

$$
\hat{J}(\xi)(u)=\left(k_{C}^{A} y^{C} \pi_{A}^{B}+x^{0} v^{A} \pi_{A}^{B}\right) \hat{s}_{B}
$$

or if $\left\{C_{B}^{A}, s^{D}\right\}$ is the basis of $\mathfrak{g}(k)^{*}$ dual to $\left\{E_{B}^{A}, s_{C}\right\}$,

$$
J(u)=\left(y^{D} \pi_{A}^{B} C_{D}^{A}+x^{0} \pi_{A}^{B} s^{A}\right) \hat{s}_{B}=\left(y_{[D} \pi_{A]}^{B} C^{A D}+x^{0} \pi_{A}^{B} s^{A}\right) \hat{s}_{B} .
$$

The function $\hat{G}$ corresponding to the metric $G$ is in $S T_{V}^{2}\left(L_{V} Y\right)$ and, via equation (5.1), produces an equivalence class of $\mathbb{R}^{1+k}$-valued Hamiltonian vector fields $\llbracket X_{\hat{G}} \rrbracket$. We choose a representative of this equivalence class, expressed in local coordinates as

$$
X_{\hat{G}}^{0}=\pi_{0}^{0} \frac{\partial}{\partial x^{0}} \quad \text { and } \quad X_{\hat{G}}^{A}=\pi_{0}^{A} \frac{\partial}{\partial x^{0}}+\iota^{B C} \pi_{B}^{A} \frac{\partial}{\partial y^{C}} .
$$

Along an integral curve of $X_{\hat{G}}^{0}$, we get the equations $\dot{x}^{0}=\pi_{0}^{0}$ and $\dot{y}^{A}=\dot{\pi}_{C}^{B}=\dot{\pi}_{0}^{D}=\dot{\pi}_{0}^{0}=0$. Integrating with suitable initial conditions we obtain $F_{\lambda}^{0}=\left(x^{0}+\pi_{0}^{0} \lambda, y^{A}, \pi_{C}^{B}, \pi_{0}^{D}, \pi_{0}^{0}\right)$. So,

$$
J^{0} \circ F_{\lambda}^{0}=J^{0}=0 .
$$

Along an integral curve of $X_{\hat{G}}^{A}$, we obtain the equations

$$
\dot{x}^{0}=\pi_{0}^{A}, \quad \dot{y}^{C}=\iota^{B C} \pi_{B}^{A}, \quad \text { and } \quad \dot{\pi}_{C}^{B}=\dot{\pi}_{0}^{D}=\dot{\pi}_{0}^{0}=0 .
$$

Integrating with initial conditions we get $F_{\lambda}^{A}=\left(x^{0}+\pi_{0}^{A} \lambda, y^{C}+\lambda \iota^{B C} \pi_{B}^{A}, \pi_{D}^{B}, \pi_{0}^{E}, \pi_{0}^{0}\right)$. So,

$$
\begin{aligned}
J^{B} \circ F_{\lambda}^{B} & =\left(x^{0}+\pi_{0}^{B} \lambda\right) \pi_{A}^{B} s^{A}+\left(y^{C}+\lambda \iota^{C E} \pi_{C}^{B}\right) \pi_{D}^{B} C_{E}^{D} \quad \text { no sum on } B \\
& =x^{0} \dot{y}^{C} \iota_{A C} s^{A}+y^{E} \dot{y}^{D} \iota_{A D} C_{E}^{A}+\lambda \dot{x}^{0} \iota_{A C} \dot{y}^{C} s^{A} \\
& =y_{[E} \dot{y}_{D]} C^{D E}+x^{0} \dot{y}^{C} \iota_{A C} s^{A}+\lambda \dot{x}^{0} \iota_{A C} \dot{y}^{C} s^{A} \\
& =J^{B}+\lambda \dot{x}^{0} \iota_{A C} \dot{y}^{C} s^{A} .
\end{aligned}
$$

Apparently (6.13) violates conservation of angular momentum along the flow, because $F_{\lambda}^{B}$ is not an integral of the motion. Closer observation reveals that the additional term describes the contribution to the angular momentum from shift of the point in $Y$ under the translational part of $\mathcal{G}(k)$. Indeed if we restrict the action to $O_{\iota}(k) \subset \mathcal{G}(k)$, then the extra 
term disappears as in equation (6.12), and we produce conserved angular momentum along flows in the spatial directions. Thus the $(n+k)$-symplectic geometry adjusts the angular momentum to accommodate a change in reference frame.

If $k=3$ and $\iota$ is the Euclidean metric on $\mathbb{R}^{3}$, then $\mathcal{G}(3)=E(3)$, the Euclidean group. Equation (6.13) may be interpreted to be the "parallel axis theorem" of classical mechanics. If $k=4$ and $\iota$ is the Lorentz metric, then $\mathcal{G}(4)=E(4)$, the Poincaré group, and 6.13) indicates how to transform 4 -angular momentum when boosting from one relativistic inertial reference frame to another.

\subsection{Time reparametrization symmetry in time-evolution mechanics}

Let $X=\mathbb{R}$ and let $Y=\mathbb{R} \times Q$, where $Q$ is a $k$-dimensional manifold. Again, coordinates on $y$ are $\left\{x^{0}=t, y^{A}\right\}$. Let $X$ have the Euclidean metric and let $Q$ have a Riemannian metric $g$. Assume a generic connection $\gamma: T Y \rightarrow V(T Y)$ expressed locally by $\gamma(v)=\left(v^{A}-v^{0} \gamma^{A}\right) \frac{\partial}{\partial y^{A}}$. The resulting Kaluza-Klein metric $G$ on $Y$ has local coordinate expression,

$$
G(v, w)=\left(1+g_{A B} \gamma^{A} \gamma^{B}\right) v^{0} w^{0}+g_{A B} \gamma^{A}\left(v^{B} w^{0}+v^{0} w^{B}\right)+g_{A B} v^{A} w^{B} .
$$

Let $\mathcal{G}=$ Diff $\mathbb{R}$ act on $Y$ by time reparametrizations. The corresponding Lie algebra $\mathfrak{g}$ may be identified with $C^{\infty}(\mathbb{R})$, and we may identify $\mathfrak{g}^{*}$, the space of densities on $\mathbb{R}$, with $\mathfrak{g}$. This action can be represented in Aut $Y$, as it leaves the fiber of $Y$ constant. Clearly this action may be lifted to $L_{V} Y$. In local coordinates, $f \in$ Diff $\mathbb{R}$ has infinitesimal generator $f_{Y}=f\left(x^{0}\right) \frac{\partial}{\partial x^{0}}$, which lifts to a projectable vector field on $L_{V} Y, f_{L_{V} Y}=f\left(x^{0}\right) \frac{\partial}{\partial x^{0}}$. Using Lemma 3.3 the corresponding momentum observable is

$$
\left.\hat{J}_{L_{V} Y}(f)=f\left(x^{0}\right) \frac{\partial}{\partial x^{0}}\right\lrcorner\left(\left(\pi_{B}^{A} d y^{B}+\pi_{0}^{A} d x^{0}\right) \hat{s}_{A}+\pi_{0}^{0} d x^{0} \hat{r}_{0}\right)=f\left(x^{0}\right)\left(\pi_{0}^{A} \hat{s}_{A}+\pi_{0}^{0} \hat{r}_{0}\right)
$$

or

$$
J_{L_{V} Y}: f \mapsto f \cdot\left(\pi_{0}^{A} \hat{s}_{A}+\pi_{0}^{0} \hat{r}_{0}\right) .
$$

On $L_{V} Y$ we may note the time-dependence of the momentum observables. In fact the second term describes momentum along the time parameter and the first term provides an adjustment in momentum in the fiber of $Y$ to account for the time reparametrization.

The lift of the action to $Z$ is $f_{Z}=f_{Y}=f\left(x^{0}\right) \frac{\partial}{\partial x^{0}}$, and its momentum observable on $Z$ is

$$
\left.\hat{J}_{Z}(f)\left(x^{0}\right)=f_{Z}\right\lrcorner \Theta=p f\left(x^{0}\right) .
$$

Note the time-dependence of the momentum observables on $Z \simeq T^{*} \mathbb{R} \times T^{*} Q$.

The tensorial function $\hat{G}$ on $L_{V} Y$ corresponding to the Kaluza-Klein metric $G$ is in $S T_{V}^{2}\left(L_{V} Y\right)$ and, by (5.1), produces equivalence classes of Hamiltonian vector fields $\llbracket X_{\hat{G}} \rrbracket^{\mu}$. If in addition to (5.1) the Hamiltonian vector fields must satisfy a "no-torsion" condition,

$$
\left.\left.X_{\hat{g}}^{\mu}\right\lrcorner X_{\hat{g}}^{\nu}\right\lrcorner i^{*} d \theta=0,
$$

then the Hamiltonian vector fields are unique and may be expressed locally as

$$
X_{\hat{g}}^{\mu}=G^{\nu \lambda} \pi_{\nu}^{\mu} \frac{\partial}{\partial Y^{\lambda}}+\Gamma_{\sigma \rho}^{\nu} G^{\kappa \rho} \pi_{\kappa}^{\mu} \pi_{\nu}^{\lambda} \frac{\partial}{\partial \pi_{\sigma}^{\lambda}}
$$


where $\left\{Y^{\mu}\right\}=\left\{x^{0}, y^{A}\right\}$ and $\Gamma_{\nu \lambda}^{\mu}$ are the Christoffel symbols of the second kind for the Levi-Civita connection defined by $G$. Note that several terms such as $\pi_{A}^{i}, \Gamma_{0 i}^{0}$, and $\Gamma_{00}^{k}$ will vanish in (6.14). From this we may write the differential equations for the integral curve of $X_{\hat{g}}^{0}$ through a point $u \in L_{V} Y$. We get (among others) a geodesic equation:

$$
\ddot{y}^{A}+\Gamma_{B C}^{A} \dot{y}^{B} \dot{y}^{C}+2 \Gamma_{B 0}^{A} \dot{y}^{B} \dot{x}^{0}+\Gamma_{00}^{A} \dot{x}^{0} \dot{x}^{0}=0,
$$

and an equation of parallel transport of vertical frames,

$$
\ddot{\pi}_{B}^{A}-\Gamma_{B D}^{C} \dot{y}^{D} \pi_{C}^{A}-\Gamma_{B D}^{0} \dot{y}^{D} \pi_{0}^{A}-\Gamma_{B 0}^{C} \dot{x}^{0} \pi_{C}^{A}-\Gamma_{B 0}^{0} \dot{x}^{0} \pi_{0}^{A}=0 .
$$

This improves upon a "parallel transport" result of Norris [16, 17], because for a nontrivial connection $\gamma$ we obtain parallel transport of frames along the time direction without needing to assume that one of the legs of the frame is tangent to the geodesic.

\section{Conclusions}

This investigation shows that the $(n+k)$-symplectic geometry of the bundle of vertically adapted linear frames $L_{V} Y$ of a field configuration bundle $Y$ extends the $n$-symplectic geometry of Norris 15, 16, 17] to provide momentum mappings for field theories. The "allowable" classical field momentum observables are a special case of these momentum mappings, generated by lifting a bundle automorphism of $Y$ to a momentum mapping on $L_{V} Y$. These momentum observables improve upon the momentum observables found in the multisymplectic geometry of Gotay, et al. [5, 6]. Furthermore, we may interpret a Kaluza-Klein metric tensor on $Y$ as the energy observable of the system and identify conserved quantities as those whose corresponding observables commute with the energy observable. Examples demonstrate conservation of the field, parameter space, and total momentum. Furthermore, the frame bundle perspective can allow for adjustments in the conservation laws to accommodate a change in inertial reference frame.

This work sets the stage for an $(n+k)$-symplectic reduction by symmetry. Reduction by symmetry in this context would address field theories with symmetry but would employ a finite-dimensional approach. Progress on the Lagrangian side of this problem is seen using the multisymplectic approach [2, 11] and in the $n$-symplectic approach [12, 18]. It is hoped that a full program of field-theoretic reduction by symmetry, analogous to cotangent bundle, Lie-Poisson, or Euler-Poincaré reduction, will emerge in the recent future.

\section{Acknowledgements}

This research was partially supported by ROA Supplements to NSF grants DMS 9802106 and DMS 9633161 at the California Institute of Technology and by the John M. Bennett, Sr., Fellowship at Trinity University. The author thanks J. E. Marsden for his assistance with obtaining financial support and for his comments. The author also thanks R. O. Fulp and L. K. Norris for their insights. 


\section{References}

[1] R. Abraham and J.E. Marsden: Foundations of Mechanics, 2nd ed., Benjamin and Cummings, Reading, Massachusetts 1978.

[2] M. Castrillón López, M., T.S. Ratiu and S. Shkoller: Reduction in principal fiber bundles: Covariant Euler-Poincaré equations. Proc. Amer. Math. Soc. 128 (2000), $2155-2164$

[3] C. Chevalley: Theory of Lie Groups, I, Princeton University Press, Princeton 1946.

[4] R.O. Fulp, J.K. Lawson and L.K. Norris: Generalized symplectic geometry as a covering theory for the Hamiltonian theories of classical particles and fields. J. Geom. Phys. 20 (1996), 195-206.

[5] M.J. Gotay: A multisymplectic framework for classical field theory and the calculus of variations, I: Covariant Hamiltonian formalism. In Mechanics, Analysis and Geometry: 200 Years after Lagrange, pp. 203-235, North Holland, Amsterdam 1991.

[6] M.J. Gotay, J. Isenberg and J.E. Marsden: Momentum Maps and Classical Relativistic Fields I, unpublished notes, 1997 (available at arXiv.org as physics/9801019).

[7] J. Kijowski: A finite-dimensional canonical formalism in the classical field theory. Commun. Math. Phys. 30 (1973), 99-128.

[8] J. Kijowski: Multiphase spaces and gauge in the calculus of variations. Bull. Acad. Sc. Polon. 22 (1974), 1219-1225.

[9] S. Kobayashi and K. Nomizu: Foundations of Differential Geometry, Vol. I, Interscience, New York 1963.

[10] J.K. Lawson: A frame bundle generalization of multisymplectic geometries. Rep. Math. Phys. 45 (2000), 183-205.

[11] J.E. Marsden, G.W. Patrick and S. Shkoller: Multisymplectic geometry, variational integrators, and nonlinear PDEs. Commun. Math. Phys. 199 (1998), 351-395.

[12] M. McLean and L. K. Norris: Covariant field theory on frame bundles of fibered manifolds. J. Math. Phys. 41 (2000), 6808-6823.

[13] J. Milnor: Remarks on infinite-dimensional Lie groups. In Relativity, Groups and Topology II, Les Houches Session XL, 1983, pp. 1008-1057, Elsevier, Amsterdam 1984.

[14] A. Nijenhuis: Jacobi-type identities for bilinear differential concomitants of certain tensor fields I. Indag. Math. 17 (1955), 390-397.

[15] L.K. Norris: Generalized symplectic geometry on the frame bundle of a manifold. In: Proc. Symp. Pure Math. 54, Part 2, pp. 435-465, Amer. Math. Soc., Providence, Rhode Island 1993. 
[16] L.K. Norris: Symplectic geometry on $T^{*} M$ derived from $n$-symplectic geometry on LM. J. Geom. Phys. 13 (1994), 51-78.

[17] L.K. Norris: Schouten-Nijenhuis brackets. J. Math. Phys. 38 (1997), 2694-2709.

[18] L.K. Norris: $n$-symplectic algebra of observables in covariant Lagrangian field theory. J. Math. Phys. 42 (2001), 4827-4845.

[19] J.A. Schouten: Über Differentialkomitanten zweier kontravarianter Grossen. Proc. Kon. Ned. Akad. Wet. Amsterdam 43 (1940), 449-452. 